\title{
Phase shielding soliton in parametrically driven systems
}

\author{
Marcel G. Clerc, ${ }^{*}$ Mónica A. Garcia-Ñustes, ${ }^{\dagger}$ and Yair Zárate \\ Departamento de Física, Facultad de Ciencias Físicas y Matemáticas, Universidad de Chile, Casilla 487-3, Santiago, Chile \\ Saliya Coulibaly $\ddagger$ \\ Laboratoire de Physique des Lasers, Atomes et Molécules, CNRS UMR No. 8523, Université des Sciences et Technologies de Lille, \\ 59655 Villeneuve d'Ascq Cedex, France
}

(Received 13 September 2012; revised manuscript received 7 February 2013; published 24 May 2013)

\begin{abstract}
Parametrically driven extended systems exhibit dissipative localized states. Analytical solutions of these states are characterized by a uniform phase and a bell-shaped modulus. Recently, a type of dissipative localized state with a nonuniform phase structure has been reported: the phase shielding solitons. Using the parametrically driven and damped nonlinear Schrödinger equation, we investigate the main properties of this kind of solution in one and two dimensions and develop an analytical description for its structure and dynamics. Numerical simulations are consistent with our analytical results, showing good agreement. A numerical exploration conducted in an anisotropic ferromagnetic system in one and two dimensions indicates the presence of phase shielding solitons. The structure of these dissipative solitons is well described also by our analytical results. The presence of corrective higher-order terms is relevant in the description of the observed phase dynamical behavior.
\end{abstract}

DOI: 10.1103/PhysRevE.87.052915

PACS number(s): $89.75 . \mathrm{Kd}, 05.45 . \mathrm{Yv}$

\section{INTRODUCTION}

Particle-type solutions or macroscopic localized states arising in systems out of equilibrium have been observed in a wide range of physical systems. Examples include magnetic materials, liquid crystals, gas discharge systems, chemical reactions, fluids, granular media, and nonlinear optics media (see [1-3] and references therein). The variety of systems exhibiting these solutions confers them a universal nature. Given their particlelike properties, one can characterize them by a family of continuous parameters such as position, amplitude, and size. A prototypical model that exhibits dissipative localized states or dissipative solitons in the quasireversible limit—systems under the assumption of small injection and dissipation of energy [4-8]—is the parametrically driven and damped nonlinear Schrödinger (PDNLS) equation $[9,10]$

$$
\partial_{t} \psi=-i \nu \psi-i|\psi|^{2} \psi-i \partial_{x x} \psi-\mu \psi+\gamma \bar{\psi},
$$

where $\psi(x, t)$ is a complex field that accounts for the envelope of the oscillation in the system under study. The variable $\bar{\psi}$ stands for the complex conjugate of $\psi ;\{x, t\}$ denote the spatial and temporal coordinates, respectively; $v$ is the detuning parameter, which is proportional to the difference between half the forcing frequency and the natural frequency of the oscillator field; $\mu$ is the damping parameter, which accounts for the energy dissipation processes; and $\gamma$ is the amplitude of the parametric forcing. It is important to note that Eq. (1) describes an oscillatory focusing medium with dispersive coupling [11] since the nonlinear and spatial coupling terms have the same sign. For $v<0, \gamma \sim \mu \ll 1$, and $\mu^{2}<\gamma^{2}<$ $v^{2}+\mu^{2}$, the PDNLS model admits analytical localized states characterized by a uniform phase and a bell-like shape for the modulus of the amplitude-uniform phase solitons

\footnotetext{
*marcel@dfi.uchile.cl

†mgarcianustes@ing.uchile.cl

${ }^{\ddagger}$ saliya.coulibaly@phlam.univ-lille1.fr
}

(UPSs) $[9,10]$. Figure1 shows a typical dissipative soliton in a polar coordinate representation $\left[\psi(x, t)=R(x, t) e^{i \varphi(x, t)}\right]$ observed in the parametrically driven and damped nonlinear Schrödinger equation.

The PDNLS model has been derived in various physical contexts. Indeed, the PDNLS equation can be deduced from the amplitude equation approach in the parametrically driven pendulum chain [12-14]. Using the same approach in the context of magnetic systems for an easy-plane ferromagnetic spin chain exposed to both a constant and a time-periodic external magnetic field perpendicular to the hard axis, the PDNLS equation was obtained by means of the Landau-LifshitzGilbert equation $[15,16]$. Additional physical scenarios where the PDNLS model can be derived include surface waves in vertically oscillating layers of water [17-19], localized structures in nonlinear lattices [20], and the Kerr-type optical parametric oscillator [21].

Recently, we have shown that the phase of dissipative solitons exhibits an unexpected dynamical behavior in the PDNLS equation [22]. More precisely, we have found that, for a large range of parameters, PDNLS soliton solutions show dynamical phase fronts that, after some transient behavior, reach a stationary state surrounding the soliton core. Due to its shieldlike phase structure, this type of dissipative localized state has been denominated a phase shielding soliton (PSS). Using the asymptotic expression for the amplitude, valid far from the core of the soliton, we have determined analytically the shape of the phase fronts and their dynamics. The above analysis allows us to characterize the different type of shieldlike structures of the phase. Performing a numerical stability analysis and using the size of the system $L$ as a control parameter, we have proved that UPS solutions lose their stability through a Andronov-Hopf bifurcation followed by the appearance of the PSS as the stable solution of the system.

The emergence of phase fronts on dissipative solitons represents an alternative perspective in the study of parametrically 


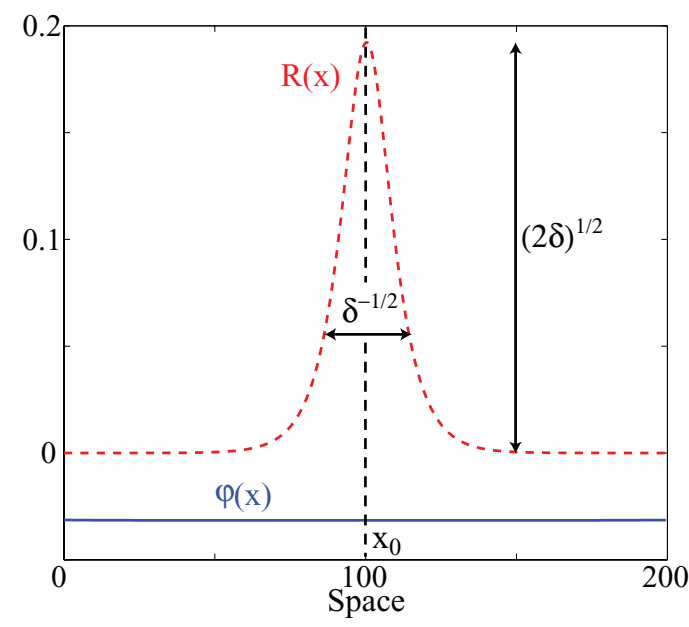

FIG. 1. (Color online) Modulus $R(x)$ (red dashed line) and phase $\phi(x)$ (blue solid line) of a UPS in the parametrically driven and damped nonlinear Schrödinger equation with $\mu=0.1000, v=$ $-0.0122, \gamma=0.1002, \delta=0.0185$, and $L=200$. Here $x_{0}$ stands for the position of the maximum of the soliton amplitude. The soliton width and height are indicated.

driven systems. Until now, the phase of single solitons was considered uniform in most of the aforementioned physical systems. However, a more complete analysis could demonstrate the existence of PSS solutions in such physical scenarios. In the present paper we extend the analytical and numerical studies of phase shielding solitons in one-dimensional (1D) and $2 \mathrm{D}$ cases. We also analyze the appearance of PSS solutions in an easy-plane ferromagnetic spin system in both 1D and 2D configurations. For this purpose, we organize the paper in the following way. In Sec. II we review the main features of the PDNLS equation and the analytical solution of the UPS and its stability. We then introduce the phase shielding soliton solutions. An analytical description of PSS solutions and their dynamics is presented. A numerical stability analysis is also performed to establish the connection between the UPS and PSS solutions. To show the existence of the PSS in physical systems, we study in Sec. III an easy-plane ferromagnetic classical spin chain exposed to an external magnetic field. The existence, stability properties, and dynamical evolution of the phase shielding solitons in two-dimensional extended systems are analyzed in Sec. IV. In Sec. V we consider a physical example of a parametrically driven system in two dimensions: a forcing magnetic layer. We conclude with a summary in Sec. VI.

\section{DISSIPATIVE SOLITONS IN THE PDNLS MODEL IN ONE DIMENSION}

For $\mu=\gamma=0$, Eq. (1) becomes the well-known nonlinear Schrödinger equation [23], which describes the envelope of an oscillatory coupled system. This model is a timereversible Hamiltonian system with the reflection symmetry $\{t \rightarrow-t, \psi \rightarrow \bar{\psi}\}$. However, the terms proportional to the energy dissipation $\mu$ and the injection $\gamma$ break this symmetry. The higher-order terms in Eq. (1) are ruled out by a scaling analysis where $\mu \ll 1, v \sim \mu \sim \gamma,|\psi| \sim \mu^{1 / 2}, \partial_{x} \sim \mu^{1 / 2}$, and $\partial_{t} \sim \mu^{1 / 2}$.
A trivial solution of Eq. (1) is the homogeneous (quiescent) state $\psi_{0}=0$. For $v<0, \psi_{0}$ becomes unstable through a subcritical stationary bifurcation at $\gamma^{2}=\mu^{2}+v^{2}$ (the Arnold tongue) [11]. Inside this region the system has three uniform solutions $\psi_{0}=0$, and $\psi_{ \pm}=\sigma \pm i \sqrt{(\mu-\gamma)(\mu+\gamma)} \sigma$, where $\sigma=\sqrt{(\gamma-\mu)\left(-v+\sqrt{\gamma^{2}-v^{2}}\right) / 2 \gamma}$. These three states merge through a pitchfork bifurcation at $\gamma^{2}=\mu^{2}+v^{2}$ when $v>0$. However, for positive detuning, $\psi_{0}$ is stable only when $\gamma<\mu$ because this state exhibits a spatial instability at $\gamma=\mu$ [24], which gives rise to a spatially periodic state with a wave number $k_{c}=\sqrt{\nu}$.

\section{A. Solitons with constant phase}

For negative detuning, the PDNLS equation exhibits localized states or dissipative solitons supported asymptotically by the quiescent state. In order to obtain the localized states, we introduce the Madelung transformation $\psi=R(x, t) e^{i \varphi(x, t)}$ in Eq. (1). Separating the imaginary and real parts, we obtain the set of equations

$$
\begin{aligned}
& \partial_{t} R=2 \partial_{x} R \partial_{x} \varphi+R \partial_{x x} \varphi-\mu R+\gamma R \cos (2 \varphi), \\
& \partial_{t} \varphi=-v-R^{2}-\frac{\partial_{x x} R}{R}+\left(\partial_{x} \varphi\right)^{2}-\gamma \sin (2 \varphi),
\end{aligned}
$$

where $R$ and $\varphi$ denote the amplitude and phase of the field $\psi$, respectively. In the parameter region $\gamma \geqslant \mu$ and $-v \pm$ $\sqrt{\gamma^{2}-\mu^{2}} \geqslant 0$, we get nontrivial steady homoclinic solutions of the form $[9,10]$

$$
\begin{gathered}
R_{s}\left(x, x_{0}\right)=\sqrt{2 \delta_{ \pm}} \operatorname{sech}\left(\sqrt{\delta_{ \pm}}\left[x-x_{0}\right]\right), \\
\cos \left(2 \varphi_{s}\right)=\mu / \gamma,
\end{gathered}
$$

where the parameter $\delta_{ \pm} \equiv-v \pm \sqrt{\gamma^{2}-\mu^{2}}$ and $x_{0}$ stands for the position of the maximum of the soliton, which will be called the core of the soliton in what follows. The modulus width and height are given by $\sqrt{2 \delta_{ \pm}}$and $1 / \sqrt{\delta_{ \pm}}$, respectively (see Fig. 1). Hence Eqs. (4) and (5) show that such states have a bell-shaped modulus and a uniform phase (UPS).

Equation (5) shows that the system can generate dissipative solitons when the injection of energy exceeds its dissipation. As a consequence of the spatial translational invariance of Eq. (1), the dissipative solitons constitute a family of states parametrized by a continuous parameter $x_{0}$ corresponding to their Goldstone mode [25]. This parameter stands for the position of the core of the localized state (see Fig. 1). In brief, the above model admits soliton solutions in the $\{\mu, \nu, \gamma\}$ region bounded by $v<0, \mu \leqslant \gamma$, and $\gamma^{2}<\mu^{2}+v^{2}$. Under these conditions, the relation $\cos \left(2 \varphi_{s}\right)=\mu / \gamma$ admits four equilibria in the interval $[\pi,-\pi]$. As a consequence of the symmetry $\psi \rightarrow-\psi$ of Eq. (1), if $\varphi_{s}$ is a solution then $\varphi_{s} \pm \pi$ is also a solution. Figure 2 illustrates this relation and the respective stability regions of the different particle-type solutions. From this figure one can infer that the localized states appear or disappear by simultaneous saddle-node bifurcations when the injection and dissipation of energy are equal $(|\gamma|=\mu)$. The stable solutions are characterized by $\operatorname{Re}(\psi) \operatorname{Im}(\psi)<0$ $[\operatorname{Re}(\psi) \operatorname{Im}(\psi)>0]$ for $\gamma>0(\gamma<0)$, thus both fields have different signs when $\gamma>0$ [15]. In contrast, the solution with a bell-shaped modulus $|\psi|$ [Eq. (4)] also appears through 


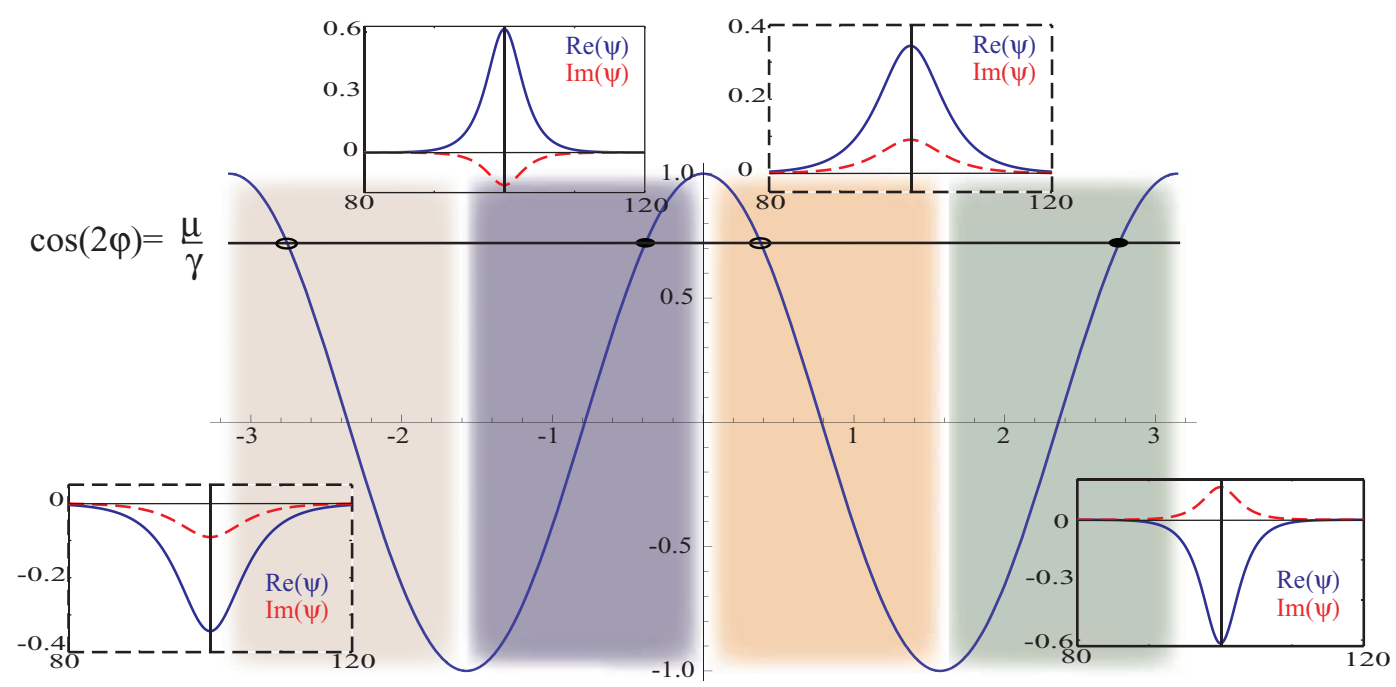

FIG. 2. (Color online) Schematic representation of the different stability regions (colored shadow) of dissipative solitons. The circles represent the different solutions of $\cos \left(2 \varphi_{s}\right)=\mu / \gamma$ : closed (open) circles correspond to stable (unstable) localized states. The insets depict the different types of dissipative solitons with $\mu=0.10, \gamma=0.13, v=-0.12$, and $L=200$, where $\delta_{+}=0.203$ and $\delta_{-}=0.037$.

a saddle-node bifurcation at $\gamma=\mu$ where $\delta_{ \pm}=-v$. The solutions of Eq. (4) with $\delta_{-}$are unstable.

\section{B. Phase shielding soliton}

Previous works have reported additional amplitude bifurcations when $v$ is increased far from the tip of the Arnold tongue where the maximum of $|\psi|$ undergoes period doubling, quasiperiodicity, and finally chaos [26]. Usually, the phase field of the dissipative soliton state is considered uniform. However, further numerical analysis reveals unexpected and rich phase dynamics of single solitons in parametrically driven systems [22], which reaches a nonuniform steady phase.

A soliton is created by slightly perturbing an initial homogenous state. The parameters are chosen to fulfill the conditions for the appearance of UPS solutions, i.e., $v<0$, $\gamma^{2}<\mu^{2}+v^{2}$, and $\mu \leqslant \gamma$. Initially, the perturbation quickly evolves to a well-defined bell-shaped amplitude. At the same time, the phase becomes uniform around the core of the localized state followed by some intricate transient that rapidly goes away from the system. The front propagation is characterized by a rather slow motion (see Fig. 3), which suddenly reaches a steady state. From this initial behavior a pair of counterpropagative fronts emerges, which propagates in a rather slow motion, reaching suddenly a steady state (see Fig. 3). Near the soliton core, the phase takes the value corresponding to the stable uniform soliton (UPS), i.e, the value $-\varphi_{s}$ or $-\varphi_{s}+\pi$. Conversely, far from the soliton position, the asymptotic values in $(-\infty, \infty)$ tend to the unstable UPS phase values either $\varphi_{s}$ or $\varphi_{s}-\pi$. Hence the number of configurations is given by different combinations of equilibrium connections (unstable-stable-unstable). There are eight stationary configurations that connect different phase equilibria. Figure 4 displays all the different phase shielding solitons. These steady phase structures depend strongly on initial conditions and are equally likely to appear in the same region of parameters.

\section{Analytical approach at dominant order}

To provide an analytical background to these phase structure dynamics, we take advantage of the $x \rightarrow-x$ symmetry of the PDNLS model [Eq. (1)]. We consider a semi-infinite domain whose origin corresponds to the core position of the soliton $x_{0}$. Numerical results show that the phase presents a single front that emerges at a position $x_{f} \gg 1 / \sqrt{\delta_{+}}$, where $x_{f}$ represents the point with the highest spatial variation of the phase. In the region $x \gg 1 / \sqrt{\delta_{+}}$, the soliton modulus $R$ decays exponentially [see Fig. 6(a)].

Defining $\Delta \equiv 1 / \sqrt{\delta_{+}}$, which accounts for the width of the soliton bell-shaped amplitude (see Fig. 1), we propose the

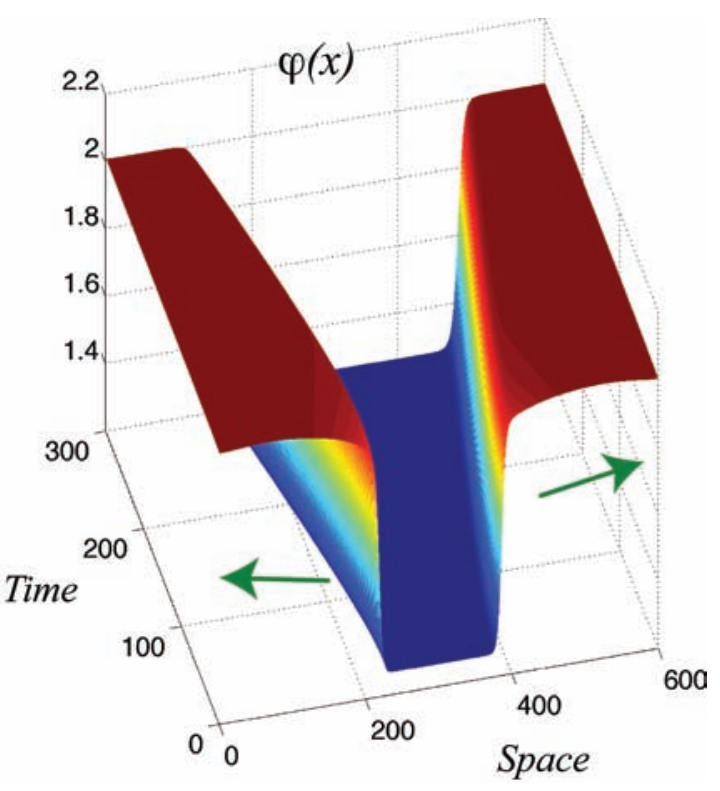

FIG. 3. (Color online) Phase propagation of the dissipative soliton. Spatiotemporal diagram of phase $\varphi(x, t)$ for $\gamma=0.123$, $\mu=0.100$, and $v=-0.093$. 

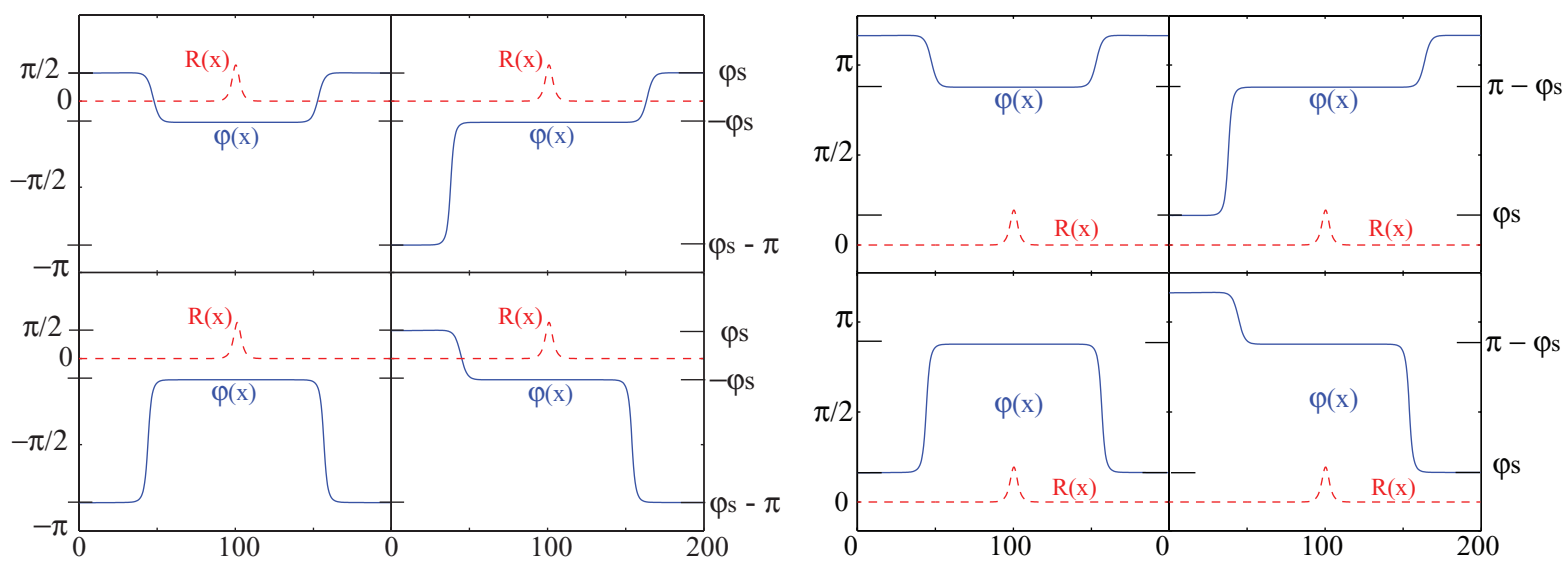

FIG. 4. (Color online) Different phase shielding soliton states in the parametrically driven damped nonlinear Schrödinger equation with $\mu=0.10, v=-0.12, \gamma=0.14$, and $L=200$. The PSS states supported by the inner uniform phases $-\varphi_{s}$ and $\pi-\varphi_{s}$ are shown on the right and left, respectively. Dashed (red) and solid (blue) lines account for the modulus and phase of the complex field $\psi$, respectively.

following ansatz for the modulus and the phase of the soliton:

$$
R\left(x \gg \Delta, x_{0}\right)=2 \sqrt{2 \delta_{+}} e^{-f\left(x, x_{0}\right)}
$$

and

$$
\varphi(x)=\varphi_{F}\left(x-x_{f}\right),
$$

respectively. At dominant order, we consider $f\left(x, x_{0}\right) \approx$ $\sqrt{\delta_{+}}\left(x-x_{0}\right)$. In this approximation, $R\left(x, x_{0}\right)$ coincides with the asymptotic exponential decay of the stable UPS modulus.

Substituting the former ansatz in (2) and (3), we obtain two different equations. The first one allows us to get analytically the dominant profile of the phase front. The second one describes the phase front dynamical behavior. Accordingly, the phase profile is characterized by

$$
\partial_{x x} \varphi_{F}=2 \sqrt{\delta_{+}} \partial_{x} \varphi_{F}+\mu-\gamma \cos \left(2 \varphi_{F}\right) .
$$

Introducing the effective potential energy $U\left(\varphi_{F}\right) \equiv-\mu \varphi_{F}+$ $(\gamma / 2) \sin \left(2 \varphi_{F}\right)$, Eq. (8) can be written as a Newton-type equation that describes a particle moving in a tilted periodic potential with an injection of energy proportional to the speed $\partial_{x} \varphi_{F}$,

$$
\partial_{x x} \varphi_{F}=-\frac{\partial U}{\partial \varphi_{F}}+2 \sqrt{\delta_{+}} \partial_{x} \varphi_{F}
$$

Hence the solutions of the above equation correspond to stationary phase fronts. The uniform equilibrium states of Eq. (8) coincide with the phase equilibria of $\cos \left(2 \varphi_{s}\right)=\mu / \gamma$ in the range from $-\pi$ to $\pi$. Therefore, the phase front solutions represent heteroclinic orbits in the $\left\{\varphi, \varphi_{x}\right\}$ space that interpolate from one equilibrium to another of the Newton-type equation (9) (see Fig. 5).

Defining the change of variable $x=2 \sqrt{\delta_{+}} x^{\prime}$ in Eq. (8), we can perform an asymptotic series $\varphi_{F}(x)=\varphi_{0}+\Gamma \varphi_{1}(x)+$ $\Gamma^{2} \varphi_{2}(x)+\cdots$, with $\Gamma \equiv 1 / 4 \delta \ll 1$, which at first order has the analytical solution

$$
\varphi_{F}\left(x, x_{f}\right) \approx \varphi_{0}= \begin{cases}f_{\mathrm{sol}}-\pi & \text { for }[-\pi,-\pi / 2) \\ f_{\mathrm{sol}} & \text { for }(-\pi / 2,-\pi / 2) \\ f_{\mathrm{sol}}+\pi & \text { for }(\pi, \pi / 2],\end{cases}
$$

where

$$
f_{\text {sol }}=\arctan \left[\sqrt{\frac{\gamma \pm \mu}{\gamma \mp \mu}} \tanh \frac{\sqrt{\gamma^{2}-\mu^{2}}\left(x-x_{f}\right)}{2 \sqrt{\delta_{+}}}\right] .
$$

Note that the phase front solutions are also parametrized by the continuous parameter $x_{f}$. Figure 6 shows the numerically computed phase front profiles, which present a difference of $1 \%$ with respect to expression (10). If one considers the dominant correction $\varphi_{F} \approx \varphi_{0}+\partial_{x} \varphi_{0} / 4 \sqrt{\delta}$, this difference decreases to $0.8 \%$.

Considering the complete soliton domain, we obtain the eighth possible shell-like configuration that we have previously observed in numerical simulations (see Fig. 4).

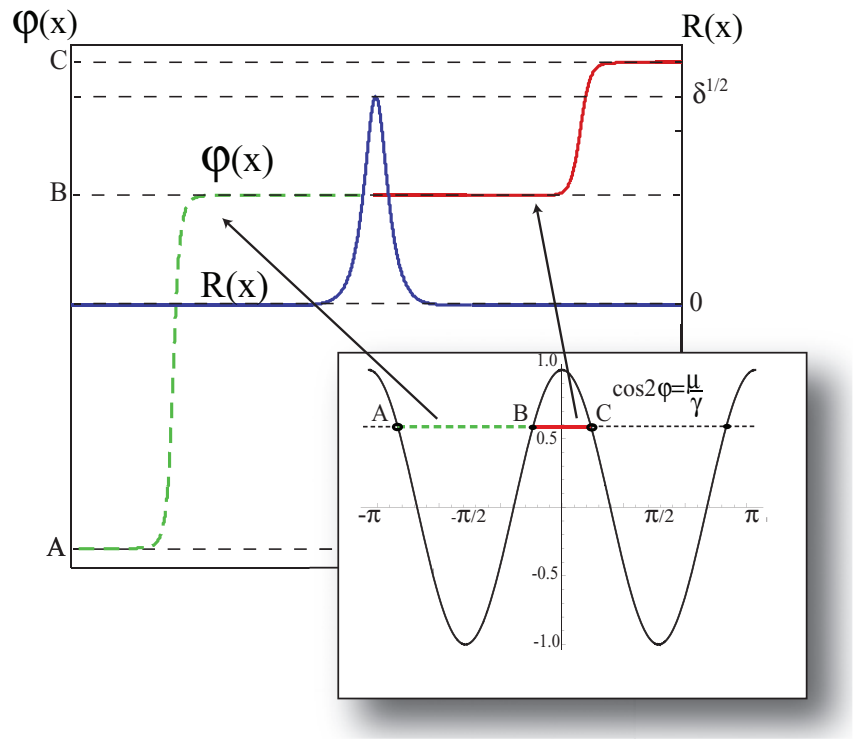

FIG. 5. (Color online) Schematic representation of the stationary phase front structure that connects different equilibrium solutions $(A-C)$ of the Newton-type equation (9). The inset is a schematic representation of the potential $U\left(\varphi_{F}\right)$. 

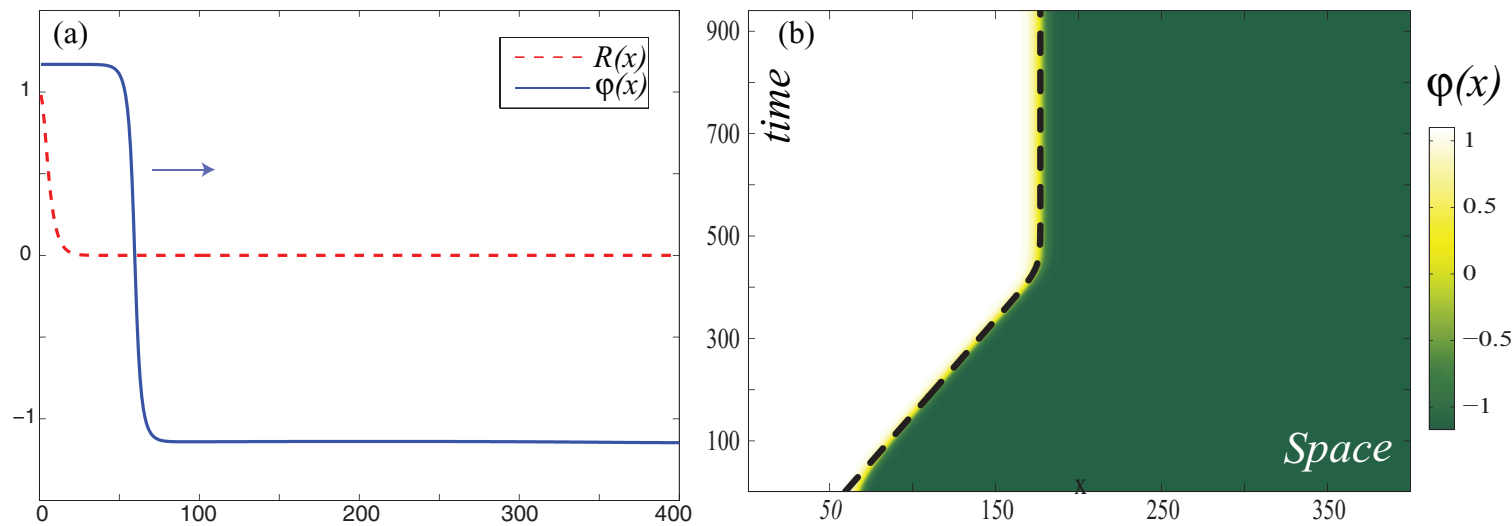

FIG. 6. (Color online) Phase propagation: (a) phase front profile considering half of the dissipative soliton and (b) spatiotemporal diagram of the phase front obtained from Eq. (1) with $\gamma=0.083, v=-0.063$, and $\mu=0.058$. The dashed curve is the numerical solution obtained using Eq. (17).

\section{Higher-order corrections}

A deeper analysis of numerical simulations reveals that PSS solutions are composed of two qualitatively different regions: inner and outer regions. The inner and outer regions stand for the central and asymptotic parts of the PSS, respectively. Note that the asymptotic phase of the PSS in the inner and outer regions coincides with the phase of the stable and unstable UPSs, respectively (see Fig. 4). Therefore, the PSS can be understood as a soliton buildup by the stable (inner region) and unstable (outer region) UPS solutions. To illustrate this statement, Fig. 7 shows the logarithm of the PSS modulus as a function of the space. Clearly, there is a crossover region

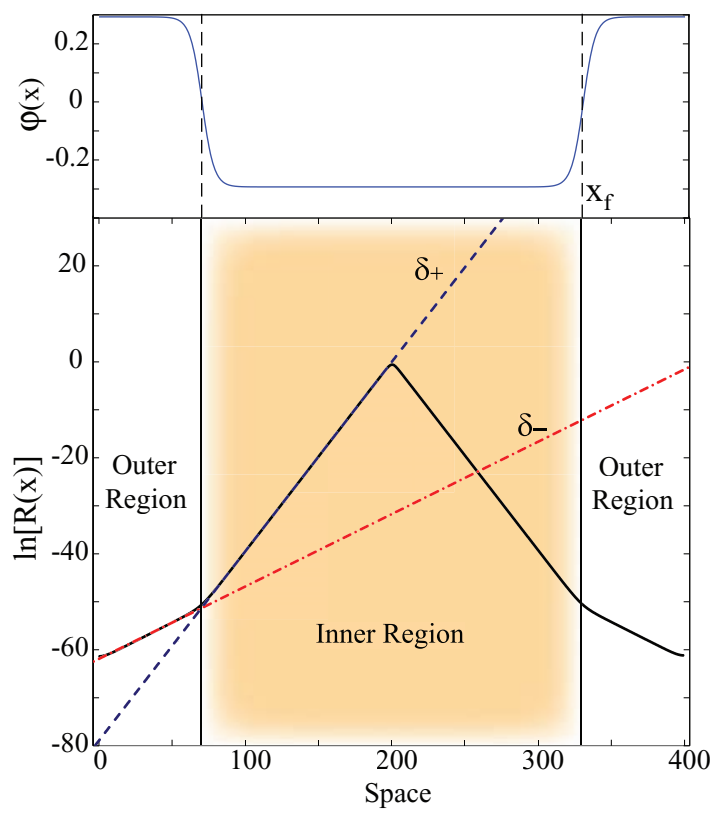

FIG. 7. (Color online) Phase $\varphi(x)$ as a function of the space (top) and logarithm of the PSS modulus $R(x)$ (bottom) with $\mu=0.1$, $v=-0.09, \gamma=0.12$, and $L=400$. Inner and outer regions are defined. The exponential decay value changes from $\sqrt{\delta_{+}}=0.3946$ (theoretical $\sqrt{\delta_{+}}=0.3954$ ) inside the inner region to $\sqrt{\delta_{-}}=0.1508$ (theoretical $\sqrt{\delta_{+}}=0.1538$ ) in the outer region. The transition point between both regions coincides with the phase front position $x_{f}$. between both exponential decay rates of the UPS solution that is characterized by a transition point. Such a point outlines the border transition between the inner and outer regions and corresponds to the phase front core position $x_{f}$. Therefore, the PSS exponential decay rate $f\left(x, x_{0}\right)$ must be amended by

$$
f\left(x, x_{0}\right) \approx \sqrt{\delta_{+}}\left(x-x_{0}\right)+B\left(x, x_{f}\right),
$$

with

$$
B\left(x, x_{f}\right) \equiv\left[\sqrt{\delta_{-}}-\sqrt{\delta_{+}}\right] \Theta\left(x-x_{f}\right)\left(x-x_{f}\right),
$$

where $\Theta\left(x-x_{f}\right)$ denotes the Heaviside function. Note that the function $f\left(x, x_{0}\right)$ is a smooth function. However, its approximation (12) is continuous but not differentiable at $x=x_{f}$.

Using the amended ansatz (12), Eqs. (8) and (9) can be reobtained in the inner and outer regions. In the inner region, Eqs. (8) and (9) remain the same. In contrast, these equations are modified by substituting the value $\delta_{+}$for $\delta_{-}$in the outer region. Following the same procedure showed in Sec. II B1, the amended front phase is obtained

$$
\varphi_{0}(x)=\arctan \left[\sqrt{\frac{\gamma \pm \mu}{\gamma \mp \mu}} \tanh \frac{\sqrt{\gamma^{2}-\mu^{2}}\left(x-x_{f}\right)}{2 \delta\left(x, x_{f}\right)}\right],
$$

with

$$
\delta\left(x, x_{f}\right) \equiv\left[\sqrt{\delta_{+}}+\left(\sqrt{\delta_{-}}-\sqrt{\delta_{+}}\right) \Theta\left(x-x_{f}\right)\right] .
$$

In this approximation the phase fronts are continuous but not differentiable at $x=x_{f}$, emphasizing that the PSS is composed of the stable and unstable UPS solutions.

It is important to note that ansatz (6) considers a uniform exponential decay rate of the modulus. Such an assumption leads, at dominant order, to the obtention of phase front solutions [Eq. (10)]. Higher-order corrections allow us to get an improved description of the phase shield soliton where the modulus also exhibits an amplitude shielding structure (see Fig. 7). However, this structure is exponentially suppressed in comparison to the soliton height $\sqrt{2 \delta_{+}}$. In contrast, the phase shielding structure is order one. Therefore, a possible experimental characterization of the PSS must be achieved by means of phase measurements. 


\section{Phase front dynamics}

As discussed in the previous section, the transient preceding the formation of the phase profile is governed by the fronts dynamics. Here we propose an analytical study of the dynamical evolution of these fronts. For this purpose let us consider the typical evolution of a soliton in a semi-infinite system, as shown in Fig. 6. As can be seen from the figure, the front displays a dynamical behavior characterized by a nontrivial motion. For the sake of simplicity, let us consider the ansatz (6) and (7) at dominant order where $f\left(x, x_{0}\right) \approx \sqrt{\delta}\left(x-x_{0}\right)$ and $\delta \equiv \delta_{+}$. Substituting in Eq. (3), we obtain the equation for position of the core

$$
-\dot{x}_{f} \partial_{x} \varphi_{F}=-(v+\delta)-8 \delta e^{-2 \sqrt{\delta} x}+\left(\partial_{x} \varphi_{F}\right)^{2}-\gamma \sin \left(2 \varphi_{F}\right) .
$$

In order to account for the front dynamics $x_{f}(t)$ has been promoted to a time-dependent function. The time derivative of $x_{f}$ is given by $\dot{x}_{f}$.

Multiplying the above equation by $\partial_{z} \varphi_{F}(z)$ with $z \equiv x-x_{f}$ and introducing the inner product $\langle f \mid g\rangle \equiv \int f g d z$, we obtain, after straightforward calculations, an ordinary differential equation for the core of the phase front

$$
\dot{x}_{f}=A+B e^{-2 \sqrt{\delta} x_{f}},
$$

where

$$
A \equiv \frac{\left\langle\left[v+\delta+\gamma \sin \left(2 \varphi_{F}\right)-\left(\partial_{z} \varphi_{F}\right)^{2}\right] \mid \partial_{z} \varphi_{F}\right\rangle}{\left\langle\partial_{z} \varphi_{F} \mid \partial_{z} \varphi_{F}\right\rangle}
$$

and

$$
B \equiv 8 \delta \frac{\left\langle e^{-2 \sqrt{\delta} z} \mid \partial_{z} \varphi_{F}\right\rangle}{\left\langle\partial_{z} \varphi_{F} \mid \partial_{z} \varphi_{F}\right\rangle}
$$

are real numbers, which can be either positive or negative, depending on the shape of the phase front. For example, when one considers a front that increases monotonically with the spatial coordinate, $A(B)$ is a negative (positive) constant. The term proportional to $A$ accounts for the constant speed at which the larger phase value invades the smaller one, giving rise to a phase front that propagates towards the position of the soliton $x_{0}$. This speed can be understood as a consequence of the effective potential energy $U\left(\varphi_{F}\right)$ difference between both equilibria. In contrast, the term proportional to $B$ accounts for the effect of spatial variation of the tail of the amplitude soliton, which induces a force that leads to phase fronts moving away from the position of the soliton. Consequently, the superposition of these two antagonistic forces generates a stable equilibrium for the position of the phase front, which is consistent with the dynamical behavior illustrated by the spatiotemporal diagram of Fig. 6(b). Solving Eq. (17), we get an analytical solution for the typical trajectory

$$
x_{f}(t)=\frac{\ln \left(\frac{B}{A}\right)}{2 \sqrt{\delta}}+\frac{\ln \left(e^{-2 \sqrt{\delta} A\left(t-t_{0}\right)}-1\right)}{2 \sqrt{\delta}}-A\left(t-t_{0}\right) .
$$

The dashed curve shown in Fig. 6(b) is obtained using the above formula wherein $A$ and $B$ are used as fitting parameters. Note that the constant $\ln (B / A) / 2 \sqrt{\delta}$ accounts for the steady equilibrium position of the front, which corresponds to the characteristic size of the shell structure in the phase. For higherorder corrections of the phase, we obtain a similar expression for the dynamics of the front.

\section{Stability analysis for the uniform phase soliton}

As we have already shown, the uniform phase and phase shielding solitons are solutions of the PDNLS model (1). Thus a natural question arises: What are the bifurcation scenarios of these solutions? Here we examine this question, performing a numerical linear stability analysis based on Ref. [15]. Given the complexity of the linear operator, an analytical stability analysis is not affordable. We consider small perturbations $\rho$ and $\Omega$ around the solutions $R_{S}(x)$ and $\varphi_{0}$, respectively, i.e.

$$
R=R_{s}(x)+\rho(x, t), \quad \varphi=\varphi_{0}+\Omega(x, t),
$$

where $\rho, \Omega \ll 1$. Substituting in (2) and (3) and linearizing, we obtain

$$
\partial_{t} \rho=2 \partial_{x} R_{s} \partial_{x} \Omega+R_{s} \partial_{x x} \Omega+2 \sqrt{\gamma^{2}-\mu^{2}} \Omega R_{s}
$$

and

$$
R_{s} \partial_{t} \Omega=\delta \rho-3 R_{s}^{2} \rho-\partial_{x x} \rho-2 \mu R_{s} \Omega,
$$

respectively. Equations (20) and (21) represent an eigenvalue problem that can be written in the matrix representation

$$
\left(\begin{array}{l}
\rho \\
\Omega
\end{array}\right)=\mathbb{M}\left(\begin{array}{l}
\rho \\
\Omega
\end{array}\right)
$$

where

$$
\mathbb{M} \equiv\left(\begin{array}{cc}
0 & 2 \partial_{x} R_{s}(x) \partial_{x}-R_{s}(x) \partial_{x x}-2 R_{s}(x) \sqrt{\gamma^{2}-\mu^{2}} \\
\frac{1}{R_{s}(x)}\left[\delta-3 R_{s}(x)^{2}-\partial_{x x}\right] & -2 \mu
\end{array}\right) .
$$

An analytical solution to Eq. (22) is a difficult task [15]. Therefore, to solve it we adopt a numerical strategy. In order to obtain the spectrum, a set of eigenvalues associated with the linear stability analysis, we proceed to discretize in space with grid points $x \rightarrow j \Delta x, F(x, t) \rightarrow F(j \Delta x, t) \equiv F_{j}(t)$ with $j=1, \ldots, N$, where $N$ is the number of points of the system and $L=N \Delta x$. In such a case, the differential operator $\mathbb{M}$ with spatiotemporal coefficients turns into a matrix of rank $2 N$. We also consider $\mu=\mu_{0}$ and $x_{0}=L / 2$ for different values of $\{\gamma, \nu\}$ in the region of existence of solitons, i.e., $\gamma^{2} \leqslant v^{2}+\mu^{2}$ and $v<0$.

The $L$ parameter controls the size effect. Changing $N$ with $\Delta x$ fixed, we can easily vary it. In previous reports, this parameter was not considered as a relevant parameter system, being usually a small constant. We shall see that the parameter $L$ plays a main role in the stability properties of dissipative states. 


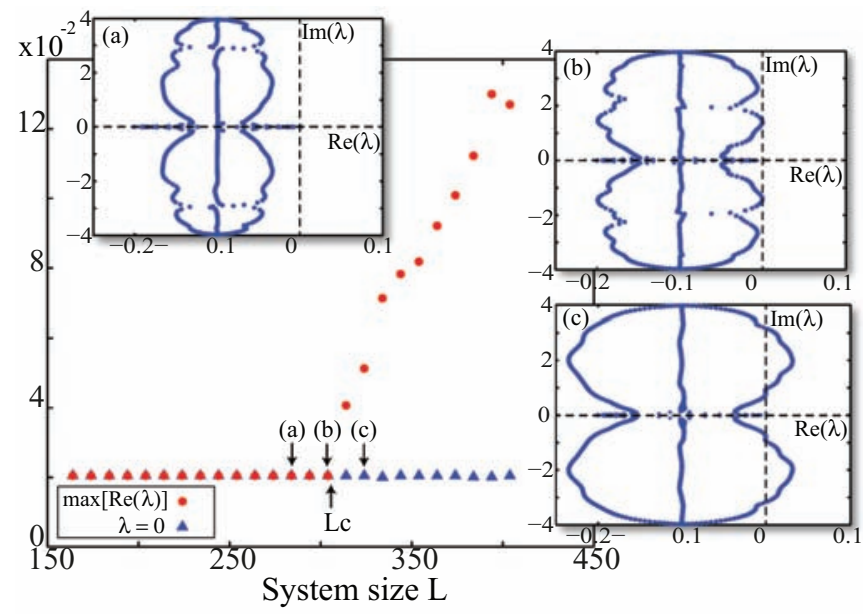

FIG. 8. (Color online) Real part of the largest eigenvalue $\max [\operatorname{Re}(\lambda)]$ (red circles) and the eigenvalue related to the Goldstone mode (blue triangles) as a function of system size. The stability of solitons is shown in the spectra of the soliton with constant phase (a) before (system size $L=284$ ), (b) during ( $L=304$ ), and (c) after $(L=324)$ the bifurcation for $\gamma=0.105, \mu=0.1$, and $v=-0.05$.

Hence let us consider $L$ as a control parameter with the system parameters $\{\mu, \nu, \gamma\}$ fixed. When $L$ is small enough the spectrum is characterized by being centered on an axis parallel to the imaginary one, where every single eigenvalue has a negative real part. Such behavior of the eigenvalues is typical of quasireversible systems [5,7]. Increasing $L$, the set of eigenvalues begins to collide, creating a continuum set. Up to a critical value of $L_{c}$, where some of them cross the imaginary axis at a nonzero frequency, the set exhibits an Andronov-Hopf bifurcation [27,28]. The inset Figs. 8(a)-8(c) outline the spectrum before, during, and after the bifurcation, respectively. The main plot of Fig. 8 illustrates the real part of the largest eigenvalue $\max [\operatorname{Re}(\lambda)]$ (red circles) and the eigenvalue related to the Goldstone mode (blue triangles) as a function of the system size $L$. As a result of the translational invariance, the eigenvalue related to the Goldstone mode is at the origin of the complex plane [25]. For $\gamma=0.105, \mu=0.1$, and $v=-0.05$, we observe that [see Fig. 8(a), inset] below the critical value $L_{c}=304$, the largest eigenvalue corresponds to the Goldstone mode. Close to the bifurcation, the largest conjugate pair of eigenvalues crosses the real axis destabilizing the uniform phase solution [see Figs. 8(b) and 8(c), insets].

The numerical stability analysis of UPS solutions reveals a strong dependence on the system size. Such a result is in accordance with the inner and outer region crossover. The inner region has a definite length for a given set of system parameters $\{\mu, \nu, \gamma\}$. If the system size is small enough ( $L$ is less than the length of the inner region), the crossover does not occur. Then the PSS solution cannot appear and the UPS is a stable solution. For $L$ greater than the length of the inner region, the UPS destabilizes, generating the PSS solution.

Given that the exponential decay of the stable UPS, and therefore the length of the inner region, is a function of the system parameters $\{\mu, \nu, \gamma\}$ it is natural to infer that by varying such parameters with $L$ fixed, the UPS destabilization will take place as well. Indeed, following the above strategy, we perform a numerical stability analysis of the UPS varying $\gamma$ for $L$ fixed

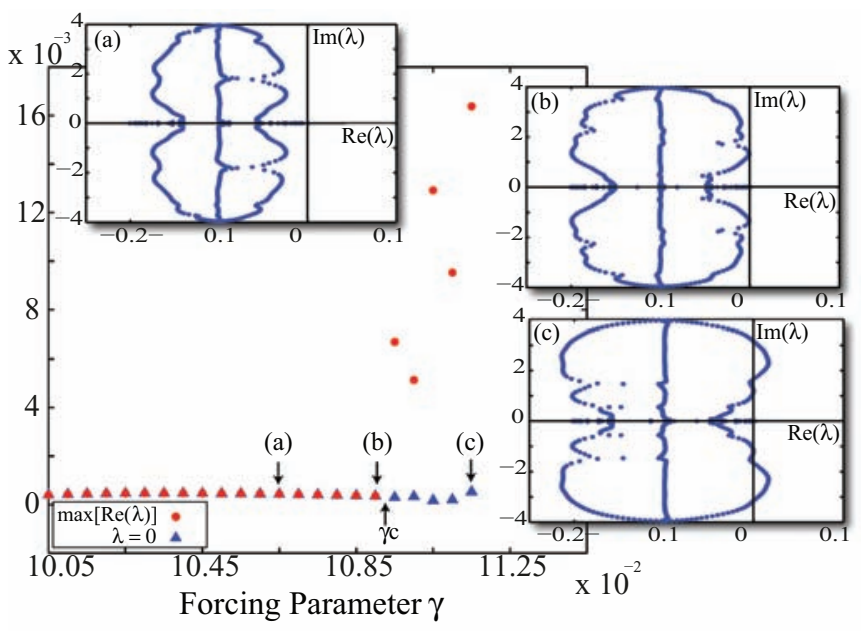

FIG. 9. (Color online) Real part of the largest eigenvalue $\max [\operatorname{Re}(\lambda)]$ (red circles) and the eigenvalue related to the Goldstone mode (blue triangles) as a function of the forcing parameter $\gamma$. The stability of solitons is shown in the spectra of the soliton with constant phase (a) before ( $\gamma=0.1065)$, (b) during ( $\gamma=0.1090)$, and (c) after $(\gamma=0.1115)$ the bifurcation for $\mu=0.1$ and $v=-0.05$ with $L=280$ fixed.

with $\mu=0.1$ and $v=-0.05$. We choose the same parameter region $\{v, \gamma\}$ with $L=280$ (before bifurcation; see Fig. 8) to ensure an initial stable UPS solution. Figure 9 displays the eigenvalue spectrum evolution as $\gamma$ varies. As before, up to a critical $\gamma_{c}$, the system exhibits an Andronov-Hopf bifurcation, which leads to the appearance of a PSS solution similar to the one observed in Figs. 8(a)-8(c).

In brief, the above instability mechanism is a robust phenomenon. Figure 10 displays the UPS stability over a wide parameter region $\{v, \gamma\}$ for $\mu=0.05$ and $L=400$ fixed. For a system size smaller than the critical one, we observe that for the parameters $0<\gamma-\mu \ll 1$, the soliton with constant phase is stable. Notwithstanding, increasing the forcing amplitude $\gamma$ or the detuning parameter $v$, the soliton becomes unstable again by an Andronov-Hopf bifurcation.

In the case that the system size is large enough, the UPS solution exhibits an Andronov-Hopf bifurcation that leads to a PSS solution. Further increasing the system parameters $\{v, \gamma\}$, a secondary bifurcation leads to a periodic soliton like those

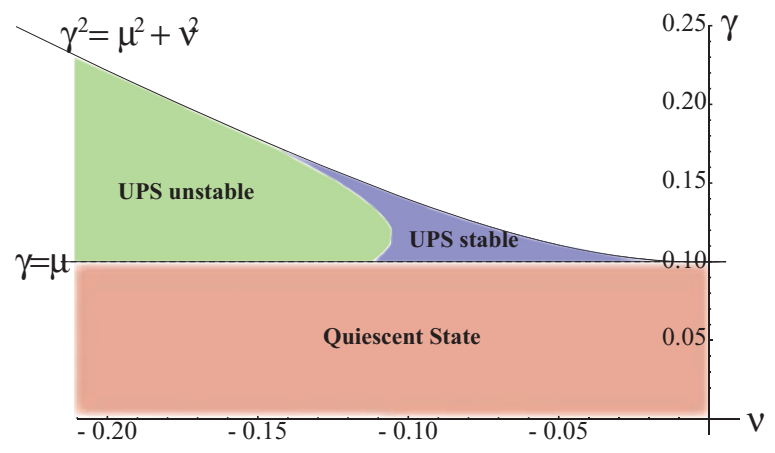

FIG. 10. (Color online) The PSS bifurcation diagram in the $\gamma-v$ space obtained by solving (22) numerically for $\mu=0.050$ and $L=$ 400. 
observed in Ref. [26]. Conversely, for small $L$ the AndronovHopf bifurcation leads directly to localized periodic solitons without a secondary one.

To verify these results, we have also performed a stability analysis of solutions in a Cartesian representation of the field $\psi$. We introduce the linear transformation $\psi=\mathcal{X}+i \mathcal{Y}$ in Eq. (1). Separating into real and imaginary parts, we obtain

$$
\begin{gathered}
\partial_{t} \mathcal{X}=\nu \mathcal{Y}+\left(\mathcal{X}^{2}+\mathcal{Y}^{2}\right) \mathcal{Y}+\partial_{x x} \mathcal{Y}-\mu \mathcal{X}+\gamma \mathcal{X}, \\
\partial_{t} \mathcal{Y}=-v \mathcal{X}-\left(\mathcal{X}^{2}+\mathcal{Y}^{2}\right) \mathcal{X}-\partial_{x x} \mathcal{X}-\mu \mathcal{Y}-\gamma \mathcal{Y},
\end{gathered}
$$

respectively. The solution for this set of equations (24) and (25) is

$$
\begin{aligned}
& \mathcal{X}_{s}=R_{s} \cos \left(\varphi_{0}\right), \\
& \mathcal{Y}_{s}=R_{s} \sin \left(\varphi_{0}\right),
\end{aligned}
$$

where $R_{s}$ and $\varphi_{0}$ are given by formulas (4) and (5) with $\delta_{+}=\delta$.

We follow the same procedure shown above and take into account small perturbations $(\delta \mathcal{X}, \delta \mathcal{Y} \ll 1)$ around the solutions $(\mathcal{X}, \mathcal{Y})$. Linearizing and using relations (4) and (5), we get the dynamical system

$$
\left(\begin{array}{l}
\dot{\delta \mathcal{X}} \\
\delta \mathcal{Y}
\end{array}\right)=\left(\begin{array}{cc}
-\mu+\gamma-R_{s}^{2} \sqrt{\gamma^{2}-\mu^{2}} / \gamma & \nu+R_{s}^{2}(2 \gamma-\mu) / \gamma+\partial_{x x} \\
-v-R_{s}^{2}(2 \gamma+\mu) / \gamma-\partial_{x x} & -\mu-\gamma+R_{s}^{2} \sqrt{\gamma^{2}-\mu^{2} / \gamma}
\end{array}\right)\left(\begin{array}{l}
\delta \mathcal{X} \\
\delta \mathcal{Y}
\end{array}\right)
$$

This system (28) yields results similar to those already observed using the polar representation.

\section{PHASE SHIELDING SOLITON IN PHYSICAL SYSTEMS}

In Sec. I we emphasized the universality of the PDNLS equation. Through an amplitude equation approach it can be shown that the equation is present in different physical systems. Based of this statement, one expects that results obtained in this context can be transposed to the original systems. In the following section we conduct numerical studies in an easyplane ferromagnetic system in order to explore the observation of PSS solutions in such a system.

\section{A. Forced magnetic wire}

Solitons in magnetism have been intensively studied in past decades due to their possible technological applications. It is known that an easy-plane ferromagnetic spin chain in the presence of both a constant and a time-periodic external magnetic field perpendicular to the hard axis exhibits localized structures. Such structures are commonly refer to as localized precession states in a forced magnetic wire. Furthermore, experimental realizations of the model have been already achieved $[29,30]$.

The forced magnetic wire is described phenomenologically by the Landau-Lifshitz-Gilbert (LLG) equation. Following an amplitude equation approach, it can be proved that, in the quasireversible limit, the system can be described by the parametrically driven and damped nonlinear Schrödinger equation [16].

Let us consider a one-dimensional anisotropic Heisenberg ferromagnetic chain formed by $N$ classical spins or a magnetic moment subject to an external magnetic field. The direction of the chain is described by the $z$ coordinate $\hat{z}=(0,0,1)$ and the external magnetic field is orthogonal to this direction, denoted by $\hat{x}=(1,0,0)$.

When the quantum effects are small enough, the vector $\mathbf{S}_{i}$ can be treated as a classical spin or a magnetic moment
[16]. According to this latter assumption, the dynamics of the magnetic moment $\mathbf{S}_{i}$ is governed by $\dot{\mathbf{S}}_{i}=-\gamma \mathbf{S}_{i} \times\left(\partial \mathcal{H} / \partial \mathbf{S}_{i}\right)$, where $\gamma$ is the gyromagnetic constant and the Hamiltonian $\mathcal{H}$ has the form

$$
\mathcal{H}=-J \sum_{i=1}^{N} \mathbf{S}_{i} \mathbf{S}_{i+1}+2 D \sum_{i=1}^{N}\left(S_{i}^{z}\right)^{2}-g \mu H_{x} \sum_{i=1}^{N} S_{i}^{x} .
$$

Here $J$ is the exchange coupling constant and $H_{x}$ and $D$ stand for the external magnetic field and the anisotropy energy, respectively.

To study the continuum limit of this set of ordinary differential equations, which accounts for a magnetic wire, we can assume that

$$
\mathbf{S}_{i}(t) \rightarrow \mathbf{S}(z, t)
$$

and

$$
\frac{J d z^{2}}{\gamma^{-1}}\left(\frac{\mathbf{S}_{i+1}-2 \mathbf{S}_{i}+\mathbf{S}_{i-1}}{d z^{2}}\right) \rightarrow l_{e x} \partial_{z}^{2} \mathbf{S}(z, t),
$$

where $l_{e x}$ denotes the characteristic interaction length. Moreover, introducing a phenomenologically dissipative source, the Gilbert damping, the motion of the magnetization field is governed by the well-known Landau-Lifshitz-Gilbert equation [31]

$$
\partial_{\tau} \mathbf{M}=\mathbf{M} \times\left[\mathbf{M}_{z z}-\beta(\mathbf{M} \cdot \hat{z})+\mathbf{H}_{e}-\alpha \partial_{\tau} \mathbf{M}\right],
$$

where $\mathbf{M} \equiv \mathbf{S} / M_{s}$ stands for the unit vector of the magnetization and $M_{s}$ is the saturation magnetization. We have also introduce the normalization $\left\{\tau \rightarrow \gamma M_{s} t, \beta \rightarrow 4 D / \gamma, \mathbf{H}_{e} \rightarrow\right.$ $\left.g \mu \mathbf{M} / \gamma M_{s}\right\}$. Here $\beta>0$ accounts for the anisotropy constant (easy-plane magnetization) and $\alpha$ the damping parameter. For several types of magnetic materials, this parameter is small [31]. When the magnetic field is time dependent, the above model (32) is a time-reversible system perturbed with injection and dissipation of energy, i.e., a quasireversible system, as long as this perturbation remains small.

As a result of the anisotropy and constant external field $\left(\mathbf{H}_{e}=H_{0} \hat{x}\right)$, the natural equilibrium of the previous model (32) corresponds to the magnetization field lying in the direction of the external magnetic field $\mathbf{M}=\hat{x}$. When spatial 

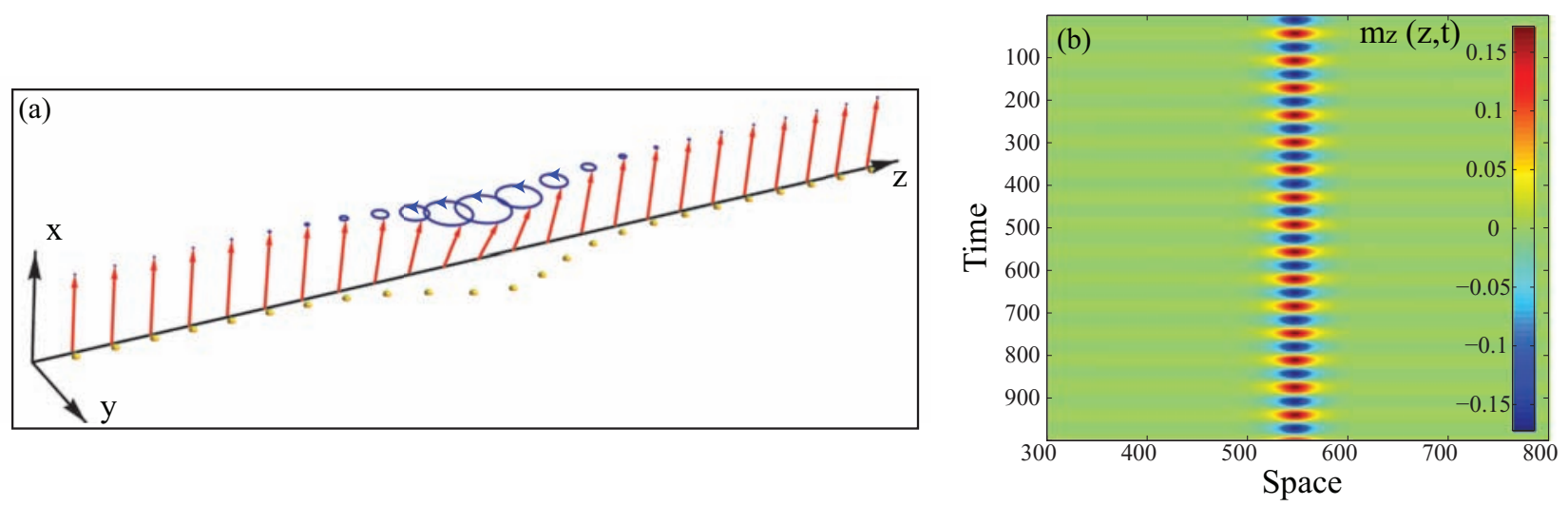

FIG. 11. (Color online) (a) Schematic representation of a soliton in a one-dimensional anisotropic ferromagnetic chain (magnetic wire). (b) Spatiotemporal evolution of a soliton for $L=1024, H_{0}=4.800, \beta=0.200, h_{1}=0.042, \alpha=0.019$, and $v=-0.030$.

coupling is ignored, it is easy to show that the dynamics around this equilibrium is described by a nonlinear oscillator with natural frequency $\omega_{0}^{2}=H_{0}\left(\beta+H_{0}\right)$ [16]. It is worth noting that in Eq. (32) the magnetization components are proportional to the external magnetic field, which therefore acts as a parametric forcing. Then if this field combines a constant and a time-periodic part $\left(\mathbf{H}_{e}=\left[H_{0}+h_{1}\right] \hat{x}\right.$, where $h_{1}=\Gamma \cos (\omega t)$ oscillates about twice the natural frequency, $\omega \equiv 2\left(\omega_{0}+v\right)$, and $v$ is the detuning parameter), the system exhibits a parametric resonance at $\Gamma^{2}\left(\beta / 4 \omega_{0}\right)^{2}=\alpha^{2}\left(H_{0}+\beta / 2\right)^{2}+v^{2}$ for small parameters $\left\{\alpha, \nu, H_{0}, \Gamma\right\}$. In the parameter space $(\Gamma, \nu)$, the region above this curve corresponds to the Arnold tongue. Dynamically speaking, this resonance corresponds to an undamped precession of the magnetization unit vector around the direction of the external magnetic field with angular velocity $\omega_{0}$ [Fig. 11(a)]. Thus, rewriting Eq. (32) for one of the components, for instance, $m_{z}$, after some calculations, considering

$$
m_{x}^{2} \approx 1-\frac{m_{y}^{2}+m_{z}^{2}}{2}
$$

and

$$
m_{y} \approx \frac{1}{H_{0}}\left[1+\frac{\Gamma}{H_{0}}\right] \dot{m}_{z},
$$

one can obtain, in the weakly nonlinear regime [16],

$$
\begin{aligned}
\ddot{m}_{z}= & -\omega_{0}^{2} m_{z}+\left(\beta+2 H_{0}\right) \partial_{z}^{2} m_{z}-\mu \dot{m}_{z}-\frac{\dot{h_{1}}}{H_{0}} \dot{m}_{z} \\
& +\left(\beta+2 H_{0}\right) h_{1} m_{z}+\frac{\beta\left(H_{0}+h_{1}\right)}{2}\left(m_{y}^{2}+m_{z}^{2}\right) m_{z},
\end{aligned}
$$

where $\mu \equiv \alpha\left(H_{0} \beta / 2\right)$ and $\gamma=\beta \Gamma / 4 \omega_{0}$ are, respectively, the effective driving strength and the detuning parameter.

Now, close to the parametric resonance, we can introduce the following ansatz $[14,16]$ into Eq. (32):

$m_{z}=4 \sqrt{\frac{\omega_{0} H_{0}}{\beta\left(\omega_{0}^{2}+3 H_{0}^{2}\right)}} \operatorname{Re}[A(z, t)] e^{i\left(\omega_{0}+v\right) t}+W(z, t, A)$,

where $W(z, t, A)$ stands for a small correction. Linearizing and imposing the solvability condition for $W(z, t, A)$, one obtains the amplitude equation of the oscillations at dominant order (the parametrically driven and damped nonlinear Schrödinger equation)

$$
\partial_{t} A=-i \nu A-i|A|^{2} A-i \partial_{Z Z} A-\mu A+\gamma \bar{A},
$$

where $Z \equiv \sqrt{2 \omega_{0} /\left(\beta+2 H_{0}\right)} z$. The terms proportional to $\{\nu, \gamma, \mu\}$ stand for the detuning, effective driving, and damping of the magnetic system.

This equation has different homogeneous states, where the simplest one is $A=0$, representing a constant magnetization along the external field direction $(\mathbf{M}=\hat{x})$. Single solitons are among the nontrivial steady states of Eq. (37) [15]. Other stationary state solutions of the PDNLS in the magnetic context can be found in Refs. [14,16,32,33].

\section{The PSS in the magnetic wire}

The chain of classical spins or magnetic wire subject to an external magnetic field represents an adequate physical system to study the formation of a phase shielding soliton. Different works have studied these magnetic localized states by means of the LLG model. Direct numerical simulations of Eq. (32) close to the Arnold tongue for negative detuning and for small values of dissipation and damping $(\mu \sim \gamma \ll 1)$ reveal the formation of a localized precession state. Figure 11(b) shows the spatiotemporal evolution of the $m_{z}$ component for this soliton.

However, phase shielding solitons are characterized for their phase structure. Therefore, we must extract information of the instantaneous phase angle $\phi_{z}$ from the knowledge of the oscillatory field $m_{z}(z, t)$. With this aim, we compute the instantaneous phase using the Hilbert transform technique for signal processing [34]. Let us recall that the original real field $m_{z}(z, t)$, can be express as

$$
m_{z}(x, t)=\operatorname{Re}\left[R(x, t) e^{i \phi(x, t)}\right],
$$

where $\operatorname{Re}(\cdot)$ represent the real part, $R(x, t)$ the modulus of the envelope, and $\phi(x, t)$ the phase.

The Hilbert transform technique reconstructs a complex variable $\theta_{z}=\theta_{r}+i \theta_{i}$ from the original real data $\theta_{r}$ and its one-sided Fourier transform $S_{r}(\omega)$,

$$
S_{r}(\omega)=\int_{-\infty}^{\infty} \theta_{r}(x, t) \Theta(\omega) e^{-i \omega t} d t,
$$




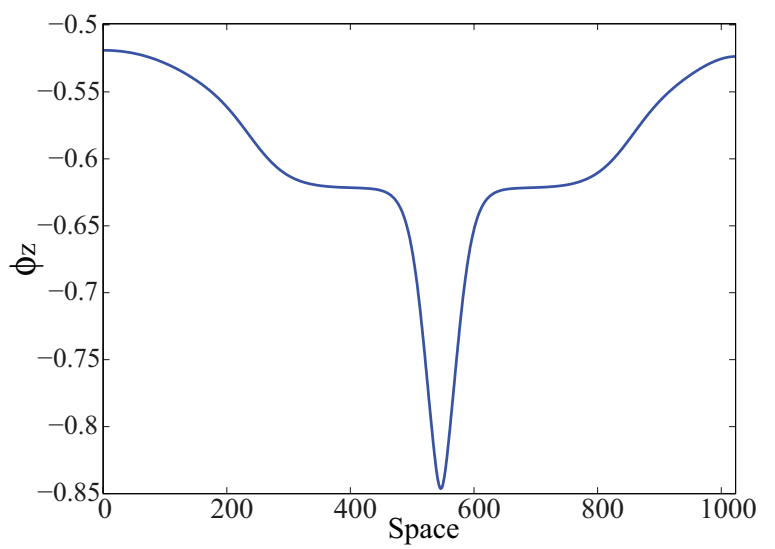

FIG. 12. (Color online) Reconstructed phase angle $\phi_{z}$ for $L=$ 1024, $H_{0}=4.800, \beta=0.200, h_{1}=0.042, \alpha=0.019$, and $v=$ -0.030 . The appearance of two opposite fronts can be observed.

where $\Theta(\omega)$ is the Heaviside step function. To obtain the onesided Fourier transformer of the imaginary part $S_{i}(\omega)$, we used the relation

$$
S_{i}(\omega)=H(\omega) S_{r}(\omega)
$$

where

$$
H(\omega)=\left\{\begin{array}{l}
-i, \quad 0 \leqslant \omega<\pi \\
i, \quad-\pi \leqslant \omega<0
\end{array}\right.
$$

is a $90^{\circ}$ phase shifter or Hilbert transformer [35].

Calculating the inverse Fourier transform of $S_{i}(\omega)$, we get the imaginary part $\theta_{i}(x, t)$ of the complex variable. Hence the modulus $R(x, t)$ and phase $\phi(x, t)$ can be easily rebuilt from the real data. This method gives a good description of the modulus and phase for band-limited signals.

Using the above described Hilbert procedure, we analyze the spatial structure of the phase at different times. Figure 12 displays the phase $\phi_{z}(x)$ at a given value $t$ for $L=1024$. The presence of two stationary but opposite fronts, quite similar to those seen in previous numerical analysis of the parametrically driven and damped nonlinear Schrödinger equation, is clear.

The phase also exhibits a slot or hole at the center of the spatial phase angle. The appearance of this hole is a consequence of the nonlinear terms present at the original model. As we show above, the amplitude equation is an approximation, at dominant order, of the original system. Higher-order corrections are not taken into account [36]. Since the simulations of the magnetic system are directly from the LLG model, they include all the nonlinear corrections. Usually such corrections are not discernible in the modulus, but become relevant in the phase, especially close to the center of the soliton. Away from the core, the nonlinear corrections decreases exponentially, hence the formation of the slot close to the center.

Another important consequence of the nonlinear corrections is related to the phase front dynamics. Numerical simulations reveal that the phase fronts reach their steady state closer to the core of the soliton than in the usual parametrically driven and damped nonlinear Schrödinger equation. Since the magnetic dissipative soliton is a precession state, the phase angle $\phi_{z}$ exhibits a modulo $2 \pi$ temporal periodicity. Therefore, there is a continuous change in the phase values
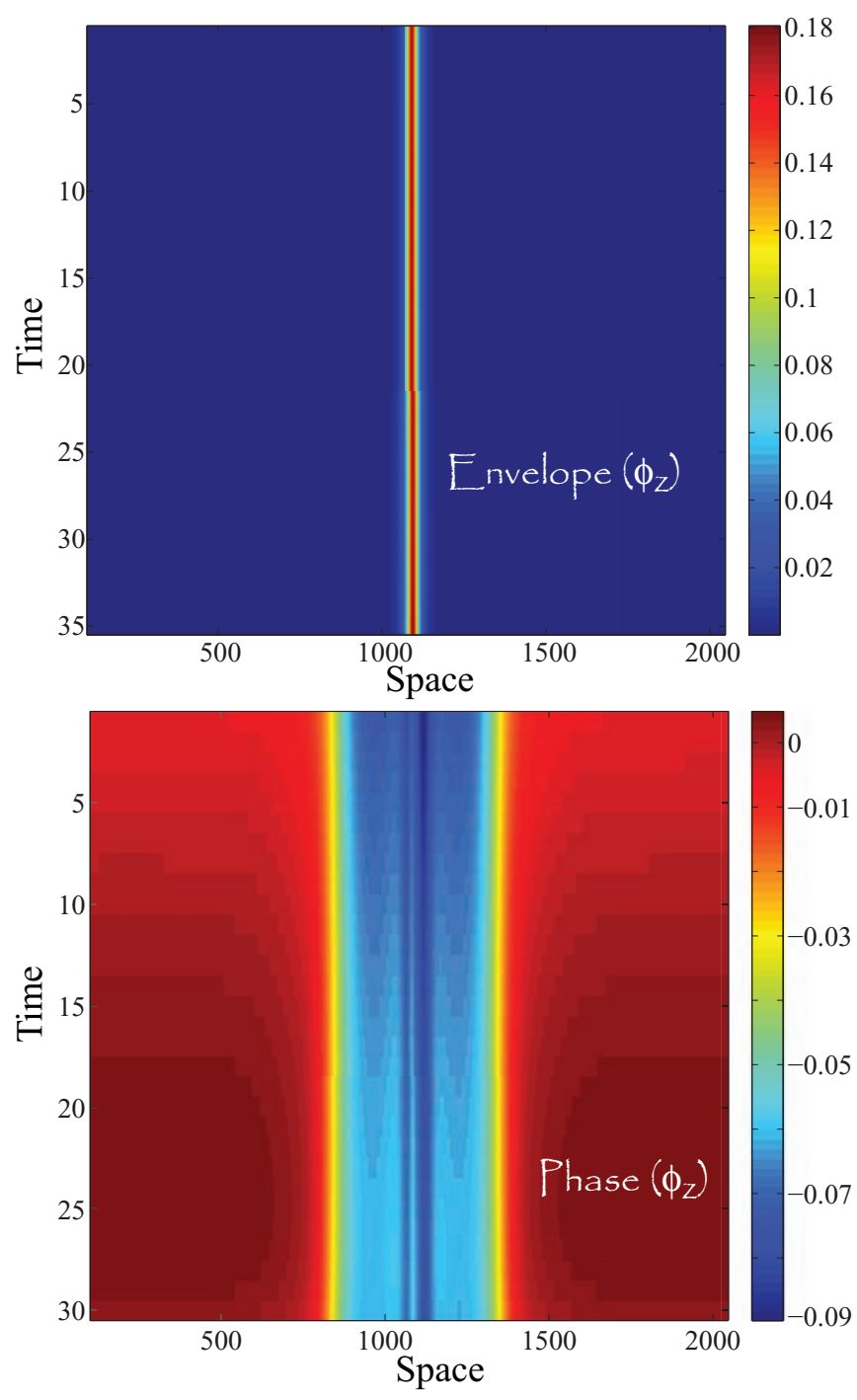

FIG. 13. (Color online) Stroboscopic spatiotemporal diagrams of the reconstructed envelope (top) and the phase angle $\phi_{z}$ (bottom) for $L=2048, H_{0}=4.800, \beta=0.200, h_{1}=0.042, \alpha=0.019$, and $v=$ -0.030 . The spatial slot displayed by the phase has been removed.

close to the ends $(0, L)$ that introduces a fictitious periodic motion of the phase front around the center. To evaluate the phase dynamical behavior and the front positions, we take stroboscopic snapshots with the same periodicity as that of the soliton oscillation. Figure 13 displays the stroboscopic phase evolution of the phase structure. It is clear that a magnetic phase shielding soliton is formed. In order to emphasize the phase fronts, we have removed the slot in the vicinity of the center. The amplitude of the envelope is shown to stress the soliton position. Different numerical simulations with $L=512,1024$, and 2048 support this observation. It is important to note that all the observed phase shielding solitons are symmetric. Asymmetric solitons have not been observed so far in the magnetic wire.

In brief, the phase fronts in the magnetic wire reach a steady state, allowing the formation of a phase shielding soliton; the shielding phase is established closer to the soliton core position, in comparison with the PSS observed in the PDNLS equation; and the phase shield structure is always symmetric 
[Fig. 4(a)]. The presence of nonlinear corrections plays an important role in the structure and dynamical behavior of the magnetic PSS.

\section{PHASE SHIELDING SOLITON IN TWO DIMENSIONS}

Localized structures presented in the previous sections are considered only in one spatial dimension. In the present section we will study the existence, stability properties, and dynamical evolution of the two-dimensional extension of the phase shielding soliton. Such extensions are not evident. Conservative solitons observed in the nonlinear Schrödinger equation in one spatial dimension collapse in two spatial dimensions [37], i.e., these solutions are unstable in two spatial dimensions. Most of the experimental observations of localized structures in parametrically driven systems have been reported in two spatial dimensions, for instance, in fluid surface waves [38], oscillons in granular media [39], and isolated states in thermal convection [40]. Note that all these observations have been realized in dissipative systems. Furthermore, the greatest difficulty in characterizing theoretically localized states in two spatial dimensions is the lack of analytical expressions of these states.

In the context of conservative systems in two spatial dimensions perturbed with energy injection through parametrically temporal modulation and dissipation-quasireversible systems [4-8] - the prototypical model is the parametrically driven and damped nonlinear Schrödinger equation

$$
\partial_{t} \psi=-i \nu \psi-i|\psi|^{2} \psi-i \nabla_{\perp}^{2} \psi-\mu \psi+\gamma \bar{\psi},
$$

where $\psi(\rho, \theta, t)$ is a complex field that accounts for the envelope of the oscillation for the system under study, $\nabla_{\perp}^{2} \equiv$ $(1 / \rho)\left[\partial_{\rho}\left(\rho \partial_{\rho}\right)\right]+\left(1 / \rho^{2}\right) \partial_{\theta \theta}$ is the Laplacian operator in polar coordinates, $\rho>0$, and $\theta \in[0,2 \pi]$. Equation (42) has been derived in two-spatial-dimensional physical systems such as the parametrically driven magnetic layer [32] and Kerr-type optical parametric oscillators [21]. In the conservative limit $(\mu=\gamma=0)$, the above equation is the nonlinear Schrödinger equation. This model is widely applied to understand wave phenomena in hydrodynamics, nonlinear optics, nonlinear acoustics, quantum condensates, heat pulses in solids, and various other nonlinear instability phenomena [41]. The nonlinear Schrödinger equation is a universal model for weakly dispersive and nonlinear media.

It is well known that Eq. (42) exhibits stable nonpropagative dissipative solitons in two spatial dimensions [42]. In contrast, in the conservative limit, Eq. (42) has unstable soliton solutions, which exhibit blowup in finite time [37]. The above phenomenon disappears in the model (42) as a result of the balance between injection and energy dissipation. In order to understand the existence, stability properties, and dynamical evolution of dissipative solitons shown by Eq. (42), let us to consider the ansatz

$$
\psi=R_{s}(\rho, t) e^{i \phi(\rho, t)} .
$$

Note that the above ansatz presents axial symmetry; that is, there is not an explicit dependence on the angle $\theta$. This assumption is based on the numerical simulations, where we do not observe significant angular dependence. Inserting (43) in Eq. (42), we obtain

$$
\begin{aligned}
\partial_{t} R_{s}= & 2 \partial_{\rho} R_{s} \partial_{\rho} \phi+\frac{R_{s}}{\rho} \partial_{\rho} \phi+R_{s} \partial_{\rho \rho} \phi-\mu R_{s} \\
& +\gamma R_{s} \cos (2 \phi), \\
R_{s} \partial_{t} \phi= & -v R_{s}-R_{s}^{3}-\frac{\partial_{\rho} R_{s}}{\rho}-\partial_{\rho \rho} R_{s}+R_{s}\left(\partial_{\rho} \phi\right)^{2} \\
& -\gamma R_{s} \sin (2 \phi) .
\end{aligned}
$$

Analogously to the one-dimensional problem, let us assume a constant phase $\phi=\phi_{0}$; thus

$$
\begin{gathered}
\cos \left(2 \phi_{0}\right)=\frac{\mu}{\gamma}, \\
\partial_{\rho \rho} R_{s}=\delta R_{s}-R_{s}^{3}-\frac{\partial_{\rho} R_{s}}{\rho},
\end{gathered}
$$

where $\delta \equiv-v+\sqrt{\gamma^{2}-\mu^{2}}$. As we have mentioned before, there is not an analytical solution of the localized state in two dimensions [42]. However, using the variational method, one can obtain a good approximation $[32,43]$

$$
R_{s}(\rho) \approx A_{0} \sqrt{\delta} \operatorname{sech}\left(B_{0} \sqrt{\frac{\delta}{2}} \rho\right),
$$

where $A_{0}=2.166$ and $B_{0}=1.32$. However, this approximation does not describe the asymptotic behavior of the dissipative soliton. The asymptotic behavior of the dissipative soliton is of the form

$$
R_{s}(\rho \rightarrow \infty) \rightarrow \frac{e^{-\sqrt{\delta} \rho}}{\sqrt{\rho}}
$$

The interaction of a pair of dissipative solitons that is of exponential type as a function of the distance between solitons has been characterized in Ref. [32]. Numerical simulations of an easy-plane ferromagnetic layer submitted to a magnetic field that combines a constant and an oscillating part show good agreement with this interaction law.

\section{A. Numerical observation of phase shielding solitons in two dimensions}

The existence of 2D solitons described by the PDNLS (42) raises the question of whether it is possible to observe a shielding phase in this case. Based on the previous simulations carried out in one dimension, we explore a similar parameter region in the $2 \mathrm{D}$ case, close to the Arnold tongue, in order to observe the possible formation of phase shielding solitons.

Indeed, we have characterized two type of phase shielding configurations. The first type consists of a phase front with axial symmetry in the range $[0,2 \pi]$. The connected uniform states are, analogously to the $1 \mathrm{D}$ case, given by the phase equilibria determined by relation (46). Figure 14 shows the typical structure of this symmetrical state around the soliton position. If we consider a 1D stationary front solution in a semi-infinite domain $(\rho>0)$, the 2D symmetric phase solution corresponds to a $2 \pi$ rotation around an axis whose origin is placed at the position of the dissipative soliton. It is important to note that the process of formation of this symmetrical state is complex since one needs special initial conditions close to the equilibrium state. 


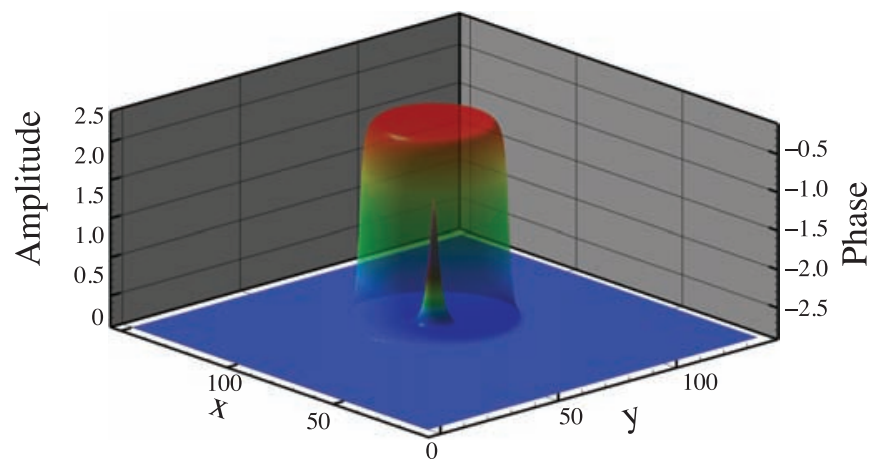

FIG. 14. (Color online) Front view of a stationary 2D symmetric phase shielding soliton observed in two-dimensional numerical simulations of the parametrically driven and damped nonlinear Schrödinger equation with $\gamma=0.560, v=-0.068$, and $\mu=0.250$. The phase and amplitude field are represented simultaneously. Colored shadow renders the phase shell-like structure $\varphi(x, y)$ that surrounds the amplitude soliton localized at the center $R(x, y)$.

The second type is characterized by a phase front axially symmetric from $[0, \pi]$ and an analogous front with different asymptotic states from $[\pi, 2 \pi]$, i.e., each phase front has semiaxial symmetry. The process of formation of this configuration starts with a well-formed 2D soliton that is slightly perturbed. After some complex phase transient state, the system exhibits the appearance of a circular phase front that spreads rather slowly. Figure 15(a) displays this primary stage. However, asymptotically, the circular structure becomes asymmetrical, giving rise to a new semicircular front that still propagates in the range $[\pi, 2 \pi]$ [see Fig. 15(b)]. Finally, the whole structure becomes stationary, creating a $2 \mathrm{D}$ asymmetric phase shielding soliton. Unlike the symmetric case, the steady phase solutions coincide only with a $\pi$ rotation around the soliton position as the center of rotation [see Fig. 15(c)]. Additionally, numerical simulations performed in a close region of parameters show the same dynamical behavior. Figure 16 give us a comparison between the stationary configuration of this shieldlike phase and the soliton size for a different set of parameter values $\{\mu, v, \gamma\}$.
It is noteworthy that this second type of two-dimensional state is characterized by being composed of all the solutions found in one dimension. Indeed, if one performs different cuts containing the center (soliton position), one can recognize the observed solutions in one dimension (see Fig. 4). Another interesting property is the following: If one calculates the phase change on a path that connects two opposite points with respect to the soliton position $\left(\int_{\Gamma} \vec{\nabla} \varphi d \vec{s}\right)$ within the region close to the position of the soliton one finds that this is zero. Nevertheless, if one takes this type of path far away from the soliton position, one finds $\int_{\Gamma^{\prime}} \vec{\nabla} \varphi d \vec{s}= \pm \pi$.

As an additional remark we would like to point out the stability of the 2D phase shielding solitons. Axially symmetric PSSs are attained only by setting up special initial conditions close to the steady state. A slight perturbation in its modulus leads to a symmetry breaking where the axial symmetry is lost and a semiaxial symmetry appears. Hence, numerically, the second type of PSS solitons has a large basin of attraction.

\section{B. Analytic approach to PSSs in two dimensions}

In this section we will discuss an analytical approach for the shielding phase solitons in two dimensions. From numerical simulations (see Fig. 16), we can observe that the phase emerges far from the soliton position. In the same manner as in Sec. II B1, to obtain the dominant phase correction we consider the exponential asymptotic decay of the modulus. Under this consideration $(\rho \rightarrow \infty)$, we use the ansatz

$$
\begin{gathered}
R_{s}(\rho \rightarrow \infty) \cong \frac{e^{-\sqrt{\delta}\left(\rho-\rho_{0}\right)}}{\sqrt{\rho}}, \\
\phi(\rho \rightarrow \infty)=\phi_{F}\left(\rho-\rho_{F}(t)\right),
\end{gathered}
$$

where $\rho_{0}$ describes the soliton position, i.e., corresponds to the position of the maximum amplitude of the soliton modulus. Moreover, we have promoted the position of the phase front core to a time-dependent function $\rho_{F}(t)$. We consider that the PSSs possess an axial symmetry for the modulus and the phase. For the sake of simplicity and without loss of generality, we can choose $\rho_{0}=0$ as the coordinate origin; at dominate order, we get a similar Newton-type equation as in the $1 \mathrm{D}$ case
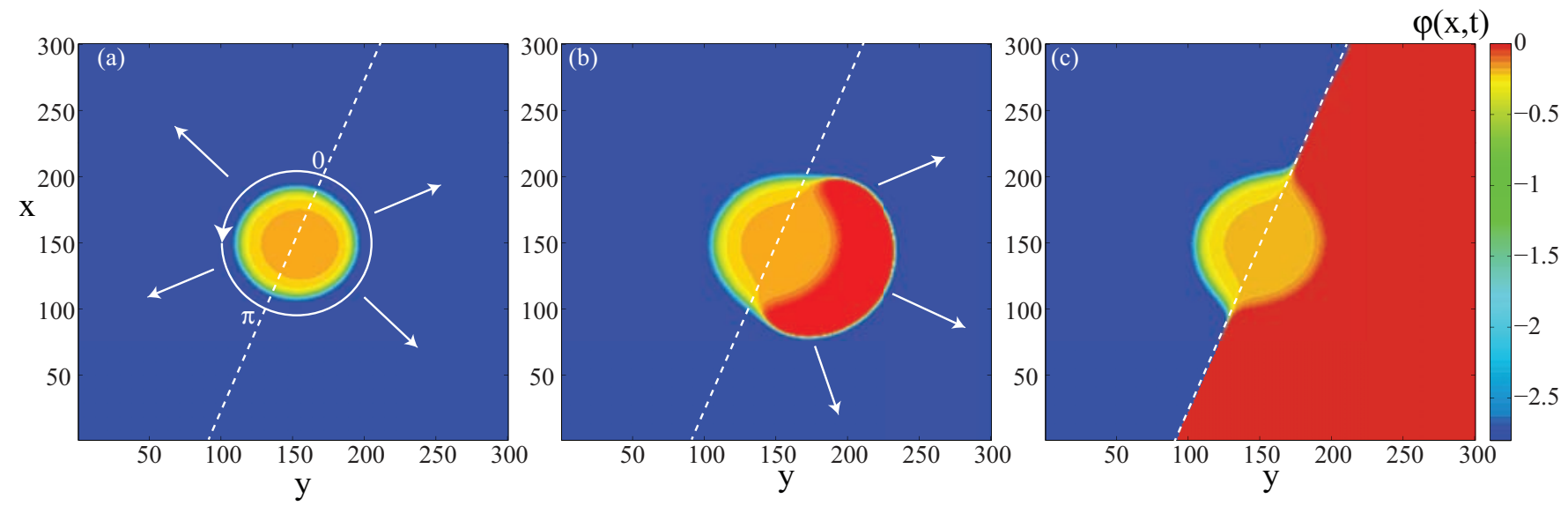

FIG. 15. (Color online) Snapshots of the phase evolution of a typical 2D PSS after a perturbation with $\gamma=0.083, v=-0.063$, and $\mu=0.058$. (a) Initially the soliton is slightly disturb. A circular front starts to propagate. (b) An additional propagative semicircular front appears. (c) The final steady configuration exhibiting a semiaxial symmetry. The dashed curve represents the $\theta$ axis. 

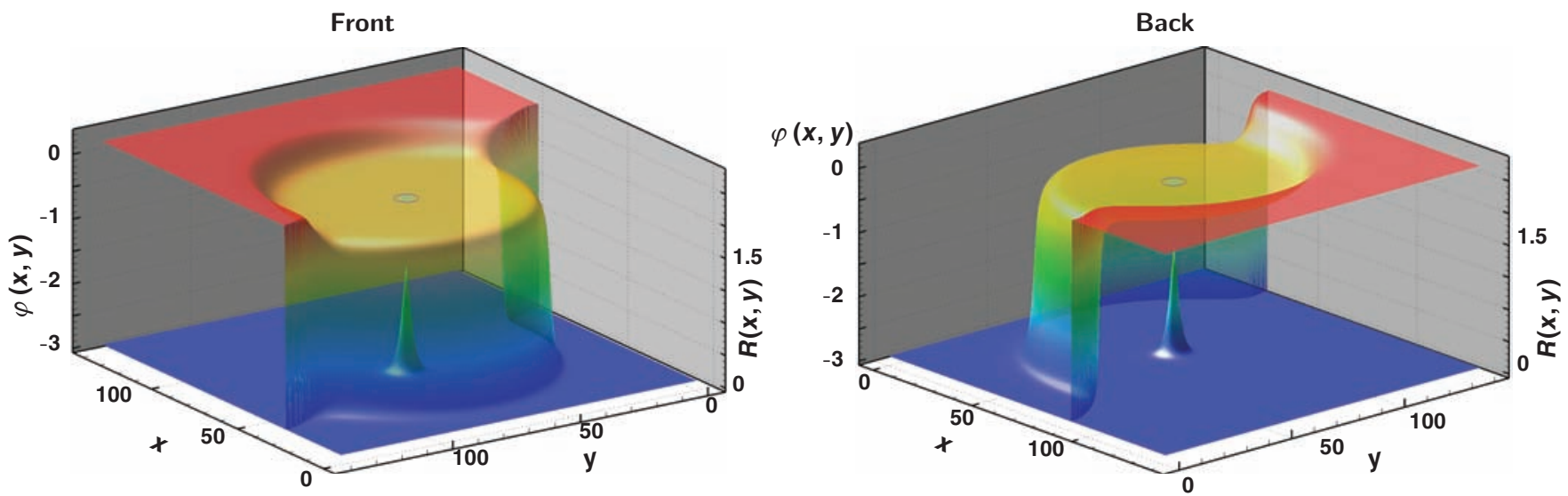

FIG. 16. (Color online) Front and back views of a stationary phase shielding soliton observed in two dimensions for the parametrically driven and damped nonlinear Schrödinger equation with $\gamma=0.140, v=-0.068$, and $\mu=0.125$. The phase and amplitude field are represented simultaneously. Colored shadow renders the phase shell-like structure $\varphi(x, y)$ that surrounds the amplitude soliton localized at the center $R(x, y)$.

$(\rho \gg 1)$

$$
\partial_{\rho \rho} \phi_{F} \approx 2 \sqrt{\delta} \partial_{\rho} \phi_{F}+\mu-\gamma \cos \left(2 \phi_{F}\right) .
$$

Thus 2D phase solutions have a front solution at dominate order of the form

$$
\phi\left(\rho, \theta, \rho_{F}\right) \approx \arctan \left[\sqrt{\frac{\gamma \pm \mu}{\gamma \mp \mu}} \tanh \frac{\sqrt{\gamma^{2}-\mu^{2}}\left(\rho-\rho_{F}\right)}{2 \sqrt{\delta}}\right] .
$$

This solution agrees with numerical simulations, as we illustrate in Fig. 17. To account analytically for the axial or semiaxial symmetry of two-dimensional PSSs, let us introduce an adequate coordinate system $\{\mathbf{r}, \theta\}$, where $\mathbf{r} \in(-\infty, \infty)$, $|\mathbf{r}|=\rho$, and $\theta \in[0, \pi)$, which is not the usual polar coordinate. Considering $R_{s} \equiv R_{s}(\mathbf{r}, t)$ and $\phi \equiv \phi(\mathbf{r}, t)$ for the semiplane domain consisting of $[0, \pi)$ and $\mathbf{r}=(0, \infty)$, we replace the ansatz (50) in (47), obtaining again Eq. (52). Therefore, we have deduced a phase front solution with the required

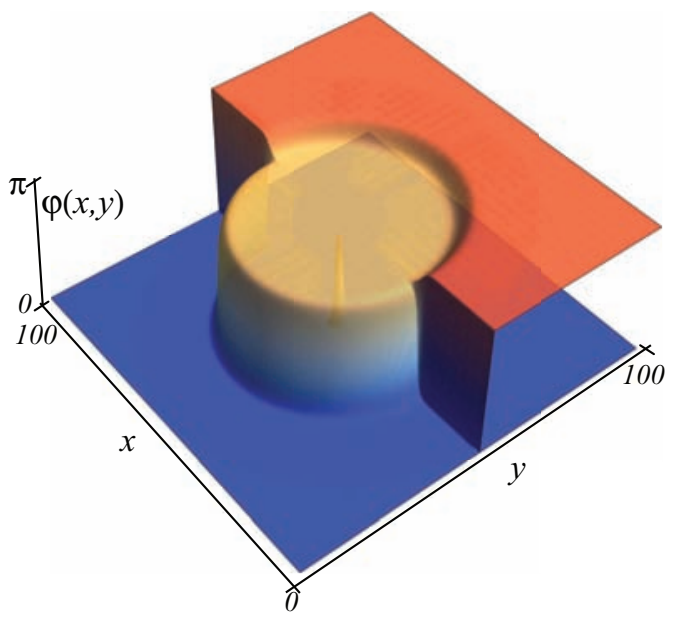

FIG. 17. (Color online) A 2D phase shielding soliton with semiaxial symmetry given at dominant order by the analytical function (53) with $\gamma=0.15, \mu=0.1$, and $v=-0.15$. The amplitude $R(x, y)$ is plotted simultaneously to illustrate the soliton position. semiaxial symmetry. Analogously to the complementary semiplane domain $\mathbf{r} \in(-\infty, 0)$ and $\theta \in[0, \pi)$, we can find the complementary phase front solution. Taking the whole domain of $\mathbf{r}$ and considering the different stationary front solutions of (52), we build up at dominant order a phase front with the asymmetrical shape observed in numerical simulations (see Fig. 16). Notice that in this approximation for PSSs with semiaxial symmetry, the interface separating the different regions is abrupt (see Fig. 17), which is a consequence of the coordinate system used to describe these solutions. The PSSs obtained numerically are smooth in this junction region (see Fig. 16).

\section{Front dynamics}

The front dynamics of phase fronts at dominant order is described by the equation obtained by replacing (50) and (51) in (45),

$$
\begin{aligned}
-\dot{\rho}_{F}(t) \partial_{z} \phi_{F}= & -(\nu+\delta)-\frac{e^{-2 \sqrt{\delta} \rho}}{\rho}+\left(\partial_{\rho} \phi_{F}\right)^{2} \\
& -\gamma \sin \left(2 \phi_{F}\right),
\end{aligned}
$$

where we have defined the appropriated comoving coordinate $z \equiv \rho-\rho_{F}$. Multiplying the above equation by $\partial_{z} \varphi_{F}$ and introducing the inner product

$$
\langle\langle f \mid g\rangle\rangle \equiv \int_{-\infty}^{\infty} \int_{0}^{\pi} f g \rho d \theta d z
$$

we obtain the following equation for the front dynamics:

$$
\dot{\rho}_{F}=\frac{\tilde{A}+A \rho_{F}+B e^{-2 \sqrt{\delta} \rho_{F}}}{C \rho_{F}},
$$

where

$$
\begin{aligned}
& \tilde{A} \equiv\left\langle\left\langle\gamma \sin \left(2 \phi_{F}\right) z \mid \partial_{\rho} \phi_{F}\right\rangle\right\rangle, \\
& A \equiv\left\langle\left\langle\left[-(\nu+\delta)+\left(\partial_{\rho} \phi_{F}\right)^{2}\right] \mid \partial_{\rho} \phi_{F}\right\rangle\right\rangle, \\
& B \equiv\left\langle\left\langle e^{-2 \sqrt{\delta} z} \mid \partial_{\rho} \phi_{F}\right\rangle\right\rangle, \quad C \equiv\left\langle\left\langle\partial_{\rho} \phi_{F} \mid \partial_{\rho} \phi_{F}\right\rangle\right\rangle .
\end{aligned}
$$

Equation (56) can be interpreted as a Newton-type equation that describes an overdamping particle in the presence of a 
force composed of three terms: a constant, a term inversely proportional to the position, and an exponential force. The constant force is responsible for spreading the phase front to the outside; in contrast, the exponential force causing the phase front propagates towards the position of the dissipative soliton in the center. The balance between these forces generates an equilibrium position for the phase front, which is consistent with the observed dynamics. At dominant order the phase dynamics is affected in two spatial dimensions by an extra term, which is inversely proportional to the distance. The exponential term is modified by a logarithmic correction. Therefore, for large distances the phase shielding soliton dynamics in two dimensions is similar to that observed in one dimension.

Similarly to Sec. II B2, we can include higher-order corrections in the amplitude of the modulus and get more accurate solutions for the structure and dynamics of phase fronts in two spatial dimensions. This allows us to understand that PSSs are composed of dissipative solitons with a different homogeneous phase (see Sec. II B2).

\section{FORCED MAGNETIC LAYER}

An easy-plane ferromagnetic layer submitted to an external parametric forcing has been analyzed within the framework of the LLG model. Such a system exhibits the formation patterns, domain walls, and localized states near a parametric resonance. By means of the amplitude equation, an interaction law of 2D localized precession states has been derived [32]. However, the magnetic layer lacks a phase numerical study for its localized structures.

Let us introduce the main features of the description of a parametrically forced magnetic layer. Let us consider an anisotropic Heisenberg ferromagnetic layer formed by $N_{x} \times N_{y}$ classical spins or magnetic moments exposed to an external magnetic field, which is contained in the plane $(x, y)$ and oriented in the direction $\hat{x} \equiv(1,0,0)$. Following the one-dimensional analysis exposed in Sec. III, we can find that the motion of the magnetization field is governed by the Landau-Lifshitz-Gilbert equation [31]

$$
\partial_{\tau} \mathbf{M}=\mathbf{M} \times\left[\nabla_{\perp}^{2} \mathbf{M}-\beta(\mathbf{M} \cdot \hat{z})+\mathbf{H}_{e}-\alpha \partial_{\tau} \mathbf{M}\right],
$$

where $\nabla_{\perp}^{2} \equiv \partial_{x x}+\partial_{y y}$ is the Laplacian operator and $\mathbf{M}=$ $\mathbf{S} / M_{S}$ stands for the unit vector of the magnetization, with $M_{s}$ the saturation magnetization. Again, we have considered a normalization of scales and parameters $\left\{\tau \rightarrow \gamma M_{s} t, \beta \rightarrow\right.$ $\left.4 D / \gamma M_{s}, \mathbf{H}_{e} \rightarrow g \mu \mathbf{H} / \gamma M_{s}\right\}$, where $\beta>0$ is the uniaxial easy-plane anisotropy constant and $\alpha$ is the damping parameter. Like the one-dimensional case, the presence of damping $\alpha>0$ and an external and constant field $\mathbf{H}_{e}=H_{0} \hat{x}$ leads to a magnetization in the direction of the external field $\mathbf{M}=\hat{x}$. When spatial coupling is ignored, it is easy to show that the dynamics around this equilibrium is described by a nonlinear oscillator with natural frequency $\omega_{0}=\sqrt{H_{0}\left(\beta+H_{0}\right)}$.

Given that the magnetization components are proportional to the external magnetic field, it acts as a parametric forcing. Applying an external magnetic field with both a constant and a time periodic part of the form $\mathbf{H}_{e}=\left[H_{0}+\Gamma \cos (\omega t)\right] \hat{x}$, oscillating about twice the natural frequency $\omega=2\left(\omega_{0}+v\right)$, where $v$ is the detuning parameter, the system exhibits a parametric resonance at $\Gamma^{2}\left(\beta / 4 \omega_{0}\right)^{2}=\alpha^{2}\left(\beta / 2+H_{0}\right)^{2}+v^{2}$ for small $\left\{v, H_{0}, \alpha, \Gamma\right\}$.

The inclusion of spatial coupling yields the formation of a localized state near the parametric resonance. To understand the dynamics of such a state, in the quasireversible limit $\Gamma \sim$ $v \sim \alpha \ll \omega_{0}$ and close to the parametric resonance, we can introduce the following ansatz into (58):

$$
\begin{aligned}
& M_{x} \approx 1-\frac{M_{y}^{2}+M_{z}^{2}}{2}, \quad M_{y} \approx \frac{1}{H_{0}}\left[1+\frac{\Gamma}{H_{0}}\right] \dot{M}_{z}, \\
& M_{z} \approx 4 \sqrt{\frac{\omega_{0} H_{0}}{\beta\left(\omega_{0}^{2}+3 H_{0}^{2}\right)}} \psi(\rho, t) e^{i\left(\omega_{0}+v\right) t}+\text { c.c. }
\end{aligned}
$$

After straightforward calculations and imposing a solvability condition for the corrections of the above ansatz, we find that the system can be described by the parametrically driven damped nonlinear Schrödinger equation

$$
\partial_{t} \psi=-i \nu \psi-i|\psi|^{2} \psi-i \nabla_{\perp}^{2} \psi-\mu \psi+\gamma \bar{\psi},
$$

with $\gamma=\beta \Gamma / 4 \omega_{0}$ and $\mu=\left(\beta / 2+H_{0}\right) \alpha$.

As we have discussed early, there is not an analytical solution for the two-dimensional PDNLS equation. However, from the approximated localized state (48), one can infer that for negative detuning, the localized breather magnetic solution appears by a saddle-node bifurcation when dissipation and energy injection are equal $(\gamma \sim \mu$ and $\nu<0)$. Furthermore, this solution is unstable when the uniform magnetization $\mathbf{M}=\hat{x}$ becomes unstable at the Arnold tongue $\left(\gamma^{2}=v^{2}+\mu^{2}\right.$ and $v<0$ ). The modulus width and height are given by $\sqrt{2 \delta}$ and $1 / \sqrt{\delta}$, respectively.

\section{A. The PSS in the magnetic layer}

Numerical simulations in appropriate parameter regions of the magnetic layer show clearly the formation of localized precession states. Previous studies have performed a numerical fit of the magnetic solution with the approximative solution (48), showing good agreement [43]. According to our analysis of the 2D PDNLS equation, an easy-plane ferromagnetic layer submitted to a external magnetic field that combines a constant and an oscillating part should present a shell-type phase structure. To capture the dynamical behavior of the phase, we perform numerical simulations of the Landau-LifshitzGilbert equation (58) over a square grid $L \times L$ for $L=512$. This system size is large enough to exhibit PSS formation (based on the results that we have obtained in one spatial dimension). Following the Hilbert transform technique, we have reconstructed the phase. Figure 18 depicts a snapshot of a localized magnetic state for $H_{0}=0.1000, \lambda=0.0100$, $\beta=1.0000, v=-0.0035$, and $\Gamma=0.0081$. As we expected, a stable shielding phase is formed surrounding the soliton. In the same figure, we show the corresponding soliton solution.

As in the 1D magnetic case, this shell-type structure is always symmetric having a $2 \pi$ rotation. A modulo $2 \pi$ periodicity is also present. The phase front also reaches its stationary state closer to the typical slot present in the center. This produces a bell-like shape as we can observed in Fig. 18. 


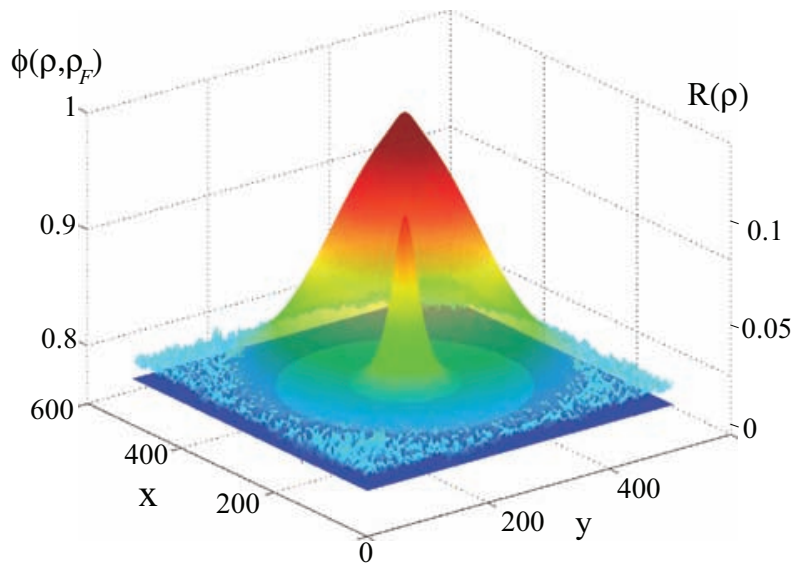

FIG. 18. (Color online) Phase shielding soliton obtained by a numerical simulation of Eq. (58) in a square magnetic plane $L \times L$ with $L=512, H_{0}=0.10000, \beta=1, h_{1}=0.00800, \alpha=0.01900$, and $v=-0.00394$. The reconstructed phase in two dimensions $\phi_{z}\left(\rho, \rho_{z}\right)$ and the amplitude field $R(\rho)$ are represented simultaneously. The bell-like shape reveals the presence of the characteristic slot, observed also in the 1D case, in the center of the plane.

In this situation, the slot is smaller that in the 1D case and it can be appreciated at the top of the phase structure.

Despite the numerical simulations revealing a stationary phase structure around a precession state-a phase shielding soliton-a deeper study is required to establish qualitatively and quantitatively the effect of the nonlinear corrections over the phase dynamics.

\section{CONCLUSION}

Parametrically forced systems exhibit oscillatory localized solutions, which are an extension to dissipative systems of conservative breathers. We have shown the emergence and characterization of unexpected phase structures surroundings the modulus of a localized state: the phase shielding soliton. Different types of localized states have been observed in numerical simulations. The appearance of each phase configuration strongly depends of initial conditions. A slight change of these conditions can lead to a different phase shielding structure using the same system parameters. However, a full comprehension of their basin of attraction as well as their stability is an open question.

Because of the universality of this phenomenon, we have described this type of oscillatory localized state in the context of the parametrically driven and damped nonlinear Schrödinger equation. Considering the asymptotic behavior of the amplitude solution away from the soliton position, we have derived an analytical expression for the front phase in a semi-infinite domain. The stationary phase front represents heteroclinic orbits in the $\{\varphi, \partial \varphi\}$ space. Such states present different types of configurations. The dynamical behavior of these fronts exhibits a nonuniform translation that is ruled by two antagonistic forces generating stable equilibria.

A deeper analysis of numerical simulations reveals that PSS solutions are composed of two qualitatively different regions: inner and outer regions. Both regions are separated by a crossover point between the exponential decay rates of the stable and the unstable UPS solution. Therefore, the PSSs are composed of both UPS solutions.

We investigate the PSS stability by means of numerical analysis. We find that the phase stability is dependent on the system size $L$. Below a critical value of $L$, the phase remains uniform, but for large enough values of it, the system destabilizes through an Andronov-Hopf bifurcation. We also show that the system also presents an instability for a given $L$, but for large values of the amplitude or the frequency of the forcing $\{\gamma, \nu\}$. Hence the shielding phase solutions exist in a wide parameter region far from the limit $\mu \sim \gamma$. Both critical values are related to the length of the inner region, i.e., to the exponential decay of the stable solution.

To confirm the presence of the shell-type phase structure in parametrically driven solitons, we have studied an anisotropic Heisenberg ferromagnetic chain formed by $N$ classical spins or magnetic moments subject to an external magnetic field. As we expected, the phase of the dissipative soliton exhibits the formation of two counterpropagative fronts, analogously to those observed for the PDNLS model.

A feature characteristic of this phase structure is the presence of a pronounced slot close to the position of dissipative soliton. Such large phase variation is a result of the nonlinear corrections of the envelope. These corrections corresponds to higher-order terms that are not taken into account in the amplitude equation approach at dominant order. Such corrections are negligible far from the position of dissipative soliton but become relevant in the phase near the soliton core.

The presence of corrective terms also affects the phase dynamics itself, as the phase front dynamics is led by an exponentially small force. This is notorious in the magnetic wire where the corrective terms cause the phase front solutions to reach their equilibrium states closer to the soliton position.

Recent studies have demonstrated that the parametrically driven and damped nonlinear Schrödinger equation does not account for all the localized structures observed in magnetic wire $[32,44]$. Consequently, the inclusion of higher order terms in the PDNLS equation is necessary for a complete description of phase front dynamics.

We have extended the phase analysis to the two-dimensional case. The parametrically driven and damped nonlinear Schrödinger equation exhibits two types of configurations: an axially symmetric state and a semiaxial symmetry composed of two semicircular fronts. The phase front dynamics is also well described by a Newtonian-type equation in the asymptotic limit of large distance $(\rho \rightarrow \infty)$. We have derived a dynamical equation that predicts a stable equilibrium position for the front and accounts for the shell-type phase structure. It is worth noting that the phase front dynamics is led again by an exponentially small force. Hence small perturbations can change radically shell-type phase structures of phase shielding solitons.

We investigate the phase behavior of oscillatory dissipative solitons in an anisotropic Heisenberg ferromagnetic layer formed by $N_{x} \times N_{y}$ classical spins or magnetic moments exposed to an external magnetic field. The numerical simulations show the existence of a complicated structure for the phase. 
The characteristic slot, observed in 1D systems, is also present in the $2 \mathrm{D}$ case. As before, the relevance of the corrective terms is evident.

The presence of corrective terms has another interesting consequence. Usually the corrective terms are related to the particular physical problem under study. The particularity of these additional terms can produce the observation of different phase phenomena for different physical systems. Therefore, we expect the observation of a variety of different phenomena in other physical systems. Still, a broader analysis of different systems, in order to establish the effect of each corrective term, is required.

In brief, close to the parametric resonance, one expects that the dynamics is well described by the parametrically driven and damped nonlinear Schrödinger equation. Indeed, this amplitude equation has been derived in several physical contexts to describe the appearance of patterns and localized states. Far from the parametric resonance, the PDNLS equation has been used as prototypical model of the dynamics. We show that the localized state exhibited by this model has an unexpected and intriguing shell-type phase structure. We expect that this type of phase behavior would be observed on several parametrically driven systems such as mechanical, optical, granular, fluid, magnetic, and chemical systems.

\section{ACKNOWLEDGMENTS}

The authors are grateful for fruitful discussions with L. Gordillo, N. Perinet, C. Falcón, and D. Laroze and to the anonymous referee for providing constructive comments and help in improving the contents of this paper. The authors acknowledge financial support from the ANR-CONICYT 39 (Grant No. ANR-2010-INTB-402-02), “Colors.” M.G.C. and M.A.G-N. are grateful for the financial support from FONDECYT Projects No. 1120320 and No. 3110024, respectively. Y.Z. acknowledges financial support from CONICYT by Beca Magister Nacional.
[1] Localized States in Physics: Solitons and Patterns, edited by O. Descalzi, M. Clerc, S. Residori, and G. Assanto (Springer, Berlin, 2011).

[2] H.-G. Purwins, H. U. Bödeker, and Sh. Amiranashvili, Adv. Phys. 59, 485 (2010).

[3] T. Ackemann, W. Firth, and G. Oppo, Adv. At. Mol. Opt. Phys. 57, 323 (2009).

[4] M. Clerc, P. Coullet, and E. Tirapegui, Int. J. Bifurcat. Chaos 11, 591 (2001).

[5] M. Clerc, P. Coullet, and E. Tirapegui, Phys. Rev. Lett. 83, 3820 (1999).

[6] M. Clerc, P. Coullet, and E. Tirapegui, Prog. Theor. Phys. Suppl. No. 139, 337 (2000).

[7] M. Clerc, P. Coullet, and E. Tirapegui, Opt. Commun. 167, 159 (1999).

[8] M. Clerc, P. Coullet, N. Vandenberghe, and E. Tirapegui, Phys. Lett. A 287, 198 (2001).

[9] J. Miles, J. Fluid Mech. 83, 153 (1977).

[10] I. V. Barashenkov and E. V. Zemlyanaya, Phys. Rev. Lett. 83, 2568 (1999).

[11] V. I. Arnold, Geometrical Methods in the Theory of Ordinary Differential Equations (Springer, New York, 1983).

[12] N. V. Alexeeva, I. V. Barashenkov, and G. P. Tsironis, Phys. Rev. Lett. 84, 3053 (2000).

[13] M. G. Clerc, S. Coulibaly, and D. Laroze, Int. J. Bifurcat. Chaos 11, 3525 (2009).

[14] M. G. Clerc, S. Coulibaly, and D. Laroze, Phys. Rev. E 77, 056209 (2008).

[15] I. Barashenkov, M. Bogdan, and V. Korobov, Europhys. Lett. 15, 113 (1991).

[16] M. G. Clerc, S. Coulibaly, and D. Laroze, Physica D 239, 72 (2010).

[17] W. Zhang and J. Viñals, Phys. Rev. Lett. 74, 690 (1995).

[18] M. Clerc, S. Coulibaly, N. Mujica, R. Navarro, and T. Sauma, Philos. Trans. R. Soc. London Ser. A 367, 3213 (2009).

[19] X. Wang and R. Wei, Phys. Rev. E 57, 2405 (1998).
[20] B. Denardo, B. Galvin, A. Greenfield, A. Larraza, S. Putterman, and W. Wright, Phys. Rev. Lett. 68, 1730 (1992).

[21] S. Longhi, Phys. Rev. E 53, 5520 (1996).

[22] M. G. Clerc, S. Coulibaly, M. A. Garcia-Ñustes, and Y. Zárate, Phys. Rev. Lett. 107, 254102 (2011).

[23] A. C. Newell, Solitons in Mathematics and Physics (Society for Industrial and Applied Mathematics, Philadelphia, 1985).

[24] P. Coullet, T. Frisch, and G. Sonnino, Phys. Rev. E 49, 2087 (1994).

[25] L. M. Pismen, Patterns and Interfaces in Dissipative Dynamics, Springer Series in Synergetics (Springer, Berlin, 2006).

[26] M. Bondila, I. V. Barashenkov, and M. M. Bogdan, Physica D 87, 314 (1995).

[27] S. H. Strogatz, Nonlinear Dynamics and Chaos: With Applications to Physics, Biology, Chemistry and Engineering (AddisonWesley, Reading, MA, 1994).

[28] J. Hale and H. Koçak, in Dynamics and Bifurcations, edited by J. E. Marsden, L. Sirovich, M. Golubitsky, W. Jäger, and F. John, Texts in Applied Mathematics, Vol. 3 (Springer, New York, 1991).

[29] A. B. Ustinov, V. E. Demidov, A. V. Kondrashov, B. A. Kalinikos, and S. O. Demokritov, Phys. Rev. Lett. 106, 017201 (2011).

[30] A. B. Ustinov, B. A. Kalinikos, V. E. Demidov, and S. O. Demokritov, Phys. Rev. B 81, 180406 (2010).

[31] G. Bertotti, I. D. Mayergoyz, and C. Serpico, Nonlinear Magnetization Dynamics in Nanosystems (Elsevier, Amsterdam, 2009).

[32] M. G. Clerc, S. Coulibaly, and D. Laroze, Europhys. Lett. 90, 38005 (2010).

[33] I. V. Barashenkov and E. V. Zemlyanaya, Phys. Rev. E 83, 056610 (2011).

[34] J. Claerbout, Fundamentals of Geophysical Data Processing (McGraw-Hill, New York, 1976).

[35] A. V. Oppenheim and R. W. Schafer, Digital Signal Processing (Prentice Hall, Englewood Cliffs, NJ, 1975). 
[36] M. Cross and H. Greenside, Pattern Formation and Dynamics in Nonequilibrium Systems (Cambridge University Press, New York, 2009).

[37] L. Berge, Phys. Rep. 303, 260 (1998).

[38] W. S. Edwards and S. Fauve, J. Fluid Mech. 278, 123 (1994).

[39] P. B. Umbanhowar, F. Melo, and H. L. Swinney, Nature (London) 382, 793 (1996).

[40] R. Heinrichs, G. Ahlers, and D. S. Cannell, Phys. Rev. A 35, 2761 (1987).
[41] C. Sulem and P.-L. Sulem, in Nonlinear Schrödinger Equations: Self-Focusing and Wave Collapse, edited by J. E. Mardsen and L. Sirovich, Applied Mathematical Sciences, Vol. 139 (Springer, New York, 1999).

[42] I. V. Barashenkov, N. V. Alexeeva, and E. V. Zemlyanaya, Phys. Rev. Lett. 89, 104101 (2002).

[43] O. Bang, Y. S. Kivshar, A. V. Buryak, A. De Rossi, and S. Trillo, Phys. Rev. E 58, 5057 (1998).

[44] M. G. Clerc, S. Coulibaly, and D. Laroze, Europhys. Lett. 97, 30006 (2012). 